\title{
A Kastler-Kalau-Walze Type Theorem for 7-Dimensional Manifolds with Boundary
}

\author{
Jian Wang ${ }^{1,2}$ and Yong Wang \\ ${ }^{1}$ School of Mathematics and Statistics, Northeast Normal University, Changchun 130024, China \\ ${ }^{2}$ School of Science, Tianjin University of Technology and Education, Tianjin 300222, China \\ Correspondence should be addressed to Yong Wang; wangy581@nenu.edu.cn
}

Received 27 June 2014; Accepted 19 August 2014; Published 14 October 2014

Academic Editor: Gang Xu

Copyright (c) $2014 \mathrm{~J}$. Wang and Y. Wang. This is an open access article distributed under the Creative Commons Attribution License, which permits unrestricted use, distribution, and reproduction in any medium, provided the original work is properly cited.

We give a brute-force proof of the Kastler-Kalau-Walze type theorem for 7-dimensional manifolds with boundary.

\section{Introduction}

The noncommutative residue found in $[1,2]$ plays a prominent role in noncommutative geometry. For one-dimensional manifolds, the noncommutative residue was discovered by Adler [3] in connection with geometric aspects of nonlinear partial differential equations. For arbitrary closed compact $n$-dimensional manifolds, the noncommutative residue was introduced by Wodzicki in [2] using the theory of zeta functions of elliptic pseudodifferential operators. In [4], Connes used the noncommutative residue to derive a conformal 4dimensional Polyakov action analogy. Furthermore, Connes made a challenging observation that the noncommutative residue of the square of the inverse of the Dirac operator was proportional to the Einstein-Hilbert action in [5]. Let $s$ be the scalar curvature and let Wres denote the noncommutative residue. Then, the Kastler-Kalau-Walze theorem gives an operator-theoretic explanation of the gravitational action and says that, for a 4-dimensional closed spin manifold, there exists a constant $c_{0}$, such that

$$
\operatorname{Wres}\left(D^{-2}\right)=c_{0} \int_{M} s \mathrm{~d} \operatorname{vol}_{M} \text {. }
$$

In [6], Kastler gave a brute-force proof of this theorem. In [7], Kalau and Walze proved this theorem in the normal coordinates system simultaneously. And then, Ackermann proved that the Wodzicki residue $\operatorname{Wres}\left(D^{-2}\right)$ in turn is essentially the second coefficient of the heat kernel expansion of $D^{2}$ in [8].

On the other hand, Fedosov et al. defined a noncommutative residue on Boutet de Monvel's algebra and proved that it was a unique continuous trace in [9]. In [10], Schrohe gave the relation between the Dixmier trace and the noncommutative residue for manifolds with boundary. For an oriented spin manifold $M$ with boundary $\partial M$, by the composition formula in Boutet de Monvel's algebra and the definition of $\widetilde{\text { Wres }}$ [11], $\widehat{\text { Wres }}\left[\left(\pi^{+} D^{-1}\right)^{2}\right]$ should be the sum of two terms from interior and boundary of $M$, where $\pi^{+} D^{-1}$ is an element in Boutet de Monvel's algebra [11]. It is well known that the gravitational action for manifolds with boundary is also the sum of two terms from interior and boundary of $M$ [12]. Considering the Kastler-Kalau-Walze theorem for manifolds without boundary, then the term from interior is proportional to gravitational action from interior, so it is natural to hope to get the gravitational action for manifolds with boundary by computing $\overparen{\text { Wres }}\left[\left(\pi^{+} D^{-1}\right)^{2}\right]$. Based on the motivation, Wang [13] proved a Kastler-Kalau-Walze type theorem for 4-dimensional spin manifolds with boundary

$$
\widetilde{\text { Wres }}\left[\left(\pi^{+} D^{-1}\right)^{2}\right]=-\frac{\Omega_{3}}{3} \int_{M} s \mathrm{~d} \mathrm{vol}_{M} \text {, }
$$

where $\Omega_{3}$ is the canonical volume of $S^{3}$. Furthermore, Wang [14] found a Kastler-Kalau-Walze type theorem for higher dimensional manifolds with boundary and generalized the 
definition of lower-dimensional volumes in [15] to manifolds with boundary. For 5-dimensional spin manifolds with boundary [14], Wang got

$$
\widetilde{\text { Wres }}\left[\left(\pi^{+} D^{-2}\right)^{2}\right]=\frac{\pi i}{2} \Omega_{2} \operatorname{vol}_{\partial M},
$$

and for 6-dimensional spin manifolds with boundary,

$$
\widetilde{\text { Wres }}\left[\left(\pi^{+} D^{-2}\right)^{2}\right]=-\frac{5 \Omega_{5}}{3} \int_{M} s \mathrm{~d} \mathrm{vol}_{M} \text {. }
$$

In order to get the boundary term, we computed the lower-dimensional volume $\mathrm{Vol}_{6}^{(1,3)}$ for 6-dimensional spin manifolds with boundary associated with $D^{-1}$ and $D^{-3}$ in [16] and obtained the volume with the boundary term

$$
\begin{aligned}
\widetilde{\text { Wres }}\left[\pi^{+} D^{-1} \cdot \pi^{+} D^{-3}\right]= & -\frac{5 \Omega_{4}}{3} \int_{M} s \mathrm{~d} \mathrm{vol}_{M} \\
& +\pi \Omega_{3} \int_{\partial M} K \mathrm{~d} \mathrm{vol}_{\partial M},
\end{aligned}
$$

where $K$ is the extrinsic curvature.

In [17], Wang proved a Kastler-Kalau-Walze type theorem for general form perturbations and the conformal perturbations of Dirac operators for compact manifolds with or without boundary. Let $M$ be 4-dimensional compact manifolds with the boundary $\partial M$ and let $\Psi$ be a general differential form on $M$, from Theorem 10 in [17]; then

$$
\begin{gathered}
\overparen{\text { Wres }}\left[\left(\pi^{+}\left(\widetilde{D}_{\Psi}\right)^{-1}\right)^{2}\right] \\
=4 \pi^{2} \int_{M} \operatorname{Tr}\left[-\frac{s}{12}-\frac{1}{2} c(\Psi) c\left(e_{i}\right) c(\Psi) c\left(e_{i}\right)\right. \\
\left.+(c(\Psi))^{2}\right] \mathrm{d} \mathrm{vol}_{M} .
\end{gathered}
$$

Recently, we computed $\overparen{\text { Wres }}\left[\pi^{+} D^{-p_{1}} \cdot \pi^{+} D^{-p_{2}}\right]$ for $n$ dimensional spin manifolds with boundary in case of $n-p_{1}-$ $p_{2} \leq 2$. In the present paper, we will restrict our attention to the case of $n-p_{1}-p_{2}=3$. We compute $\overparen{\text { Wres }}\left[\left(\pi^{+} D^{-2}\right)^{2}\right]$ for 7 -dimensional manifolds with boundary. Our main result is as follows.

Main Theorem. The following identity for 7-dimensional manifolds with boundary holds:

$$
\begin{aligned}
& \widetilde{\text { Wres }}\left[\left(\pi^{+} D^{-2}\right)^{2}\right] \\
& =\frac{\pi^{4}}{48} \int_{\partial_{M}}\left(-\frac{47}{2} K^{2}-\left.49 s_{M}\right|_{\partial_{M}}-\frac{77}{4} s_{\partial_{M}}\right) \mathrm{d} \operatorname{vol}_{\partial_{M}},
\end{aligned}
$$

where $s_{M}$ and $s_{\partial_{M}}$ are, respectively, scalar curvatures on $M$ and $\partial_{M}$. Compared with the previous results, up to the extrinsic curvature, the scalar curvature on $\partial_{M}$ and the scalar curvature on $M$ appear in the boundary term. This case essentially makes the whole calculations more difficult, and the boundary term is the sum of fifteen terms. As in computations of the boundary term, we will consider some new traces of multiplication of Clifford elements. And the inverse 4-order symbol of the Dirac operator and higher derivatives of -1-order and -3-order symbols of the Dirac operators will be extensively used.

This paper is organized as follows. In Section 2, we define lower-dimensional volumes of compact Riemannian manifolds with boundary. In Section 3, for 7-dimensional spin manifolds with boundary and the associated Dirac operators, we compute $\overparen{\text { Wres }}\left[\left(\pi^{+} D^{-2}\right)^{2}\right]$ and get a KastlerKalau-Walze type theorem in this case.

\section{Lower-Dimensional Volumes of Spin Manifolds with Boundary}

In this section, we consider an $n$-dimensional oriented Riemannian manifold $\left(M, g^{M}\right)$ with boundary $\partial_{M}$ equipped with a fixed spin structure. We assume that the metric $g^{M}$ on $M$ has the following form near the boundary:

$$
g^{M}=\frac{1}{h\left(x_{n}\right)} g^{\partial M}+d x_{n}^{2}
$$

where $g^{\partial M}$ is the metric on $\partial M$. Let $U \subset M$ be a collar neighborhood of $\partial M$ which is diffeomorphic $\partial M \times[0,1)$. By the definition of $h\left(x_{n}\right) \in C^{\infty}([0,1))$ and $h\left(x_{n}\right)>0$, there exists $\widetilde{h} \in C^{\infty}((-\varepsilon, 1))$ such that $\left.\widetilde{h}\right|_{[0,1)}=h$ and $\widetilde{h}>0$ for some sufficiently small $\varepsilon>0$. Then, there exists a metric $\widehat{g}$ on $\widehat{M}=M \bigcup_{\partial M} \partial M \times(-\varepsilon, 0]$ which has the form on $U \bigcup_{\partial M} \partial M \times(-\varepsilon, 0]$

$$
\widehat{g}=\frac{1}{\widetilde{h}\left(x_{n}\right)} g^{\partial M}+d x_{n}^{2}
$$

such that $\left.\widehat{g}\right|_{M}=g$. We fix a metric $\widehat{g}$ on the $\widehat{M}$ such that $\left.\widehat{g}\right|_{M}=g$.

Let us give the expression of Dirac operators near the boundary. Set $\widetilde{E}_{n}=\partial / \partial x_{n}$ and $\widetilde{E}_{j}=\sqrt{h\left(x_{n}\right)} E_{j}(1 \leq j \leq n-1)$, where $\left\{E_{1}, \ldots, E_{n-1}\right\}$ are orthonormal basis of $T \partial_{M}$. Let $\nabla^{L}$ denote the Levi-Civita connection about $g^{M}$. In the local coordinates $\left\{x_{i} ; 1 \leq i \leq n\right\}$ and the fixed orthonormal frame $\left\{\widetilde{E}_{1}, \ldots, \widetilde{E}_{n}\right\}$, the connection matrix $\left(\omega_{s, t}\right)$ is defined by

$$
\nabla^{L}\left(\widetilde{E}_{1}, \ldots, \widetilde{E}_{n}\right)^{t}=\left(\omega_{s, t}\right)\left(\widetilde{E}_{1}, \ldots, \widetilde{E}_{n}\right)^{t}
$$

The Dirac operator is defined by

$$
D=\sum_{j=1}^{n} c\left(\widetilde{E_{j}}\right)\left[\widetilde{E_{j}}+\frac{1}{4} \sum_{s, t} \omega_{s, t}\left(\widetilde{E_{j}}\right) c\left(\widetilde{E_{s}}\right) c\left(\widetilde{E_{t}}\right)\right]
$$

By Lemma 6.1 in [18] and Propositions 2.2 and 2.4 in [19], we have the following lemma. 
Lemma 1. Let $f=(1 / \sqrt{h})$ and $\widetilde{M}=I \times_{f} M$ be a Riemannian manifold with the metric $g_{f}=d x_{n}^{2}+f^{2}\left(x_{n}\right) g$. For vector fields $X$ and $Y$ in $\mathscr{L}(M)$, then

$$
\begin{aligned}
& \text { (1) } \widetilde{\nabla}_{\partial_{x_{n}}} \partial_{x_{n}}=0 ; \\
& \text { (2) } \widetilde{\nabla}_{\partial_{x_{n}}} X=\widetilde{\nabla}_{X} \partial_{x_{n}}=(\operatorname{lnf})^{\prime} X ; \\
& \text { (3) } \nabla_{X} Y=\nabla_{X}^{M} Y-\frac{g(X, Y)}{f} \operatorname{grad}(\mathrm{f}) .
\end{aligned}
$$

Denote $A_{j s}^{t}=2\left\langle\nabla_{E_{j}}^{L, \partial_{M}} E_{s}, E_{t}\right\rangle$; then we obtain the following lemma.

Lemma 2. The following identity holds:

$$
\begin{aligned}
& \text { (1) }\left\langle\nabla_{\widetilde{E}_{i}}^{L} \partial_{x_{n}}, \widetilde{E}_{j}\right\rangle=-\frac{h^{\prime}}{2 h} ; \\
& \text { (2) }\left\langle\nabla_{\widetilde{E}_{i}}^{L} \widetilde{E}_{j}, \partial_{x_{n}}\right\rangle=\frac{h^{\prime}}{2 h} ; \\
& \text { (3) }\left\langle\nabla_{\widetilde{E}_{j}}^{L} \widetilde{E}_{s}, \widetilde{E}_{t}\right\rangle=\frac{\sqrt{h}}{2} A_{j s}^{t} .
\end{aligned}
$$

\section{Others are zeros.}

By Lemma 2, we have the following definition.

Definition 3. The following identity holds in the coordinates near the boundary:

$$
\begin{aligned}
D= & \sum_{\beta=1}^{n} c\left(\widetilde{E_{\beta}}\right) \widetilde{E_{\beta}}-\frac{h^{\prime}}{h} c\left(d x_{n}\right) \\
& +\frac{\sqrt{h}}{8} \sum_{s, \alpha, \beta<n} A_{\beta s}^{\alpha} c\left(\widetilde{E_{\beta}}\right) c\left(\widetilde{E_{s}}\right) c\left(\widetilde{E_{\alpha}}\right) .
\end{aligned}
$$

To define the lower-dimensional volume, some basic facts and formulae about Boutet de Monvel's calculus which can be found in Section 2 in [11] are needed.

Denote by

$$
F: L^{2}\left(\mathbf{R}_{t}\right) \longrightarrow L^{2}\left(\mathbf{R}_{v}\right) ; \quad F(u)(v)=\int e^{-i v t} u(t) \mathrm{d} t
$$

the Fourier transformation and $\Phi\left(\overline{\mathbf{R}^{+}}\right)=r^{+} \Phi(\mathbf{R})$ (similarly, define $\Phi\left(\overline{\mathbf{R}^{-}}\right)$), where $\Phi(\mathbf{R})$ denotes the Schwartz space and

$$
\begin{aligned}
r^{+}: C^{\infty}(\mathbf{R}) & \longrightarrow C^{\infty}\left(\overline{\mathbf{R}^{+}}\right) ; \quad f \longrightarrow f \mid \overline{\mathbf{R}^{+}} \\
\overline{\mathbf{R}^{+}} & =\{x \geq 0 ; x \in \mathbf{R}\} .
\end{aligned}
$$

We define $H^{+}=F\left(\Phi\left(\overline{\mathbf{R}^{+}}\right)\right)$and $H_{0}^{-}=F\left(\Phi\left(\overline{\mathbf{R}^{-}}\right)\right)$which are orthogonal to each other. We have the following property: $h \in$ $H^{+}\left(H_{0}^{-}\right)$iff $h \in C^{\infty}(\mathbf{R})$ which has an analytic extension to the lower (upper) complex half-plane $\{\operatorname{Im} \xi<0\} \quad(\{\operatorname{Im} \xi>0\})$ such that, for all nonnegative integers $l$,

$$
\frac{d^{l} h}{d \xi^{l}}(\xi) \sim \sum_{k=1}^{\infty} \frac{d^{l}}{d \xi^{l}}\left(\frac{c_{k}}{\xi^{k}}\right)
$$

as $|\xi| \rightarrow+\infty, \operatorname{Im} \xi \leq 0(\operatorname{Im} \xi \geq 0)$.

Let $H^{\prime}$ be the space of all polynomials and let $H^{-}=$ $H_{0}^{-} \oplus H^{\prime} ; H=H^{+} \oplus H^{-}$. Denote by $\pi^{+}\left(\pi^{-}\right)$, respectively, the projection on $H^{+}\left(H^{-}\right)$. For calculations, we take $H=$ $\widetilde{H}=$ \{rational functions having no poles on the real axis $\}$ ( $\widetilde{H}$ is a dense set in the topology of $H$ ). Then, on $\widetilde{H}$,

$$
\pi^{+} h\left(\xi_{0}\right)=\frac{1}{2 \pi i} \lim _{u \rightarrow 0^{-}} \int_{\Gamma^{+}} \frac{h(\xi)}{\xi_{0}+i u-\xi} \mathrm{d} \xi,
$$

where $\Gamma^{+}$is a Jordan close curve which included $\operatorname{Im} \xi>0$ surrounding all the singularities of $h$ in the upper half-plane and $\xi_{0} \in \mathbf{R}$. Similarly, define $\pi^{\prime}$ on $\widetilde{H}$ :

$$
\pi^{\prime} h=\frac{1}{2 \pi} \int_{\Gamma^{+}} h(\xi) \mathrm{d} \xi .
$$

So, $\pi^{\prime}\left(H^{-}\right)=0$. For $h \in H \bigcap L^{1}(R), \pi^{\prime} h=(1 / 2 \pi) \int_{R} h(v) \mathrm{d} v$ and for $h \in H^{+} \bigcap L^{1}(R), \pi^{\prime} h=0$.

Let $M$ be an $n$-dimensional compact oriented manifold with boundary $\partial M$. Denote by $\mathscr{B}$ Boutet de Monvel's algebra; we recall the main theorem in [9].

Theorem 4 (Fedosov-Golse-Leichtnam-Schrohe). Let $X$ and $\partial X$ be connected, let $\operatorname{dim} X=n \geq 3$, and let $A=\left(\underset{T}{\pi^{+} P+G} \underset{S}{K}\right) \epsilon$ $\mathscr{B}$, and denote by $p, b$, and s the local symbols of $P, G$, and $S$, respectively. Define

$$
\begin{aligned}
\widetilde{\text { Wres }}(A)= & \int_{X} \int_{\mathrm{S}} \operatorname{tr}_{E}\left[p_{-n}(x, \xi)\right] \sigma(\xi) \mathrm{d} x \\
& +2 \pi \int_{\partial X} \int_{S^{\prime}}\left\{\operatorname{tr}_{E}\left[\left(\operatorname{tr} b_{-n}\right)\left(x^{\prime}, \xi^{\prime}\right)\right]\right. \\
& \left.+\operatorname{tr}_{F}\left[s_{1-n}\left(x^{\prime}, \xi^{\prime}\right)\right]\right\} \sigma\left(\xi^{\prime}\right) \mathrm{d} x^{\prime} .
\end{aligned}
$$

Then, (a) $\widehat{\operatorname{Wres}}([A, B])=0$, for any $A, B \in \mathscr{B}$; (b) it is a unique continuous trace on $\mathscr{B} / \mathscr{B}^{-\infty}$.

Let $p_{1}$ and $p_{2}$ be nonnegative integers and let $p_{1}+p_{2} \leq n$. Then, by Section 2.1 of [13], we have the following definition.

Definition 5. Lower-dimensional volumes of spin manifolds with boundary are defined by

$$
\operatorname{Vol}_{n}^{\left(p_{1}, p_{2}\right)} M:=\overparen{\text { Wres }}\left[\pi^{+} D^{-p_{1}} \cdot \pi^{+} D^{-p_{2}}\right] .
$$

Denote by $\sigma_{l}(A)$ the $l$-order symbol of an operator $A$. An application of (2.1.4) in [11] shows that

$$
\begin{aligned}
& \widetilde{\text { Wres }}\left[\pi^{+} D^{-p_{1}} \cdot \pi^{+} D^{-p_{2}}\right] \\
& =\int_{M} \int_{|\xi|=1} \operatorname{trace}_{S(\mathrm{TM})}\left[\sigma_{-n}\left(D^{-p_{1}-p_{2}}\right)\right] \sigma(\xi) \mathrm{d} x+\int_{\partial M} \Phi,
\end{aligned}
$$


where

$$
\begin{aligned}
\Phi=\int_{\left|\xi^{\prime}\right|=1} \int_{-\infty}^{+\infty} \sum_{j, k=0}^{\infty} \sum \frac{(-i)^{|\alpha|+j+k+1}}{\alpha !(j+k+1) !} \operatorname{trace}_{S(\mathrm{TM})} \\
\times\left[\partial_{x_{n}}^{j} \partial_{\xi^{\prime}}^{\alpha} \partial_{\xi_{n}}^{k} \sigma_{r}^{+}\left(D^{-p_{1}}\right)\left(x^{\prime}, 0, \xi^{\prime}, \xi_{n}\right)\right. \\
\quad \times \partial_{x^{\prime}}^{\alpha} \partial_{\xi_{n}}^{j+1} \partial_{x_{n}}^{k} \sigma_{l}\left(D^{-p_{2}}\right) \\
\left.\times\left(x^{\prime}, 0, \xi^{\prime}, \xi_{n}\right)\right] \mathrm{d} \xi_{n} \sigma\left(\xi^{\prime}\right) \mathrm{d} x^{\prime}
\end{aligned}
$$

and the sum is taken over $r-k+|\alpha|+\ell-j-1=-n, r \leq-p_{1}$, $\ell \leq-p_{2}$.

\section{A Kastler-Kalau-Walze Type Theorem for 7- Dimensional Spin Manifolds with Boundary}

In this section, we compute the lower-dimensional volume for 7-dimensional compact manifolds with boundary and get a Kastler-Kalau-Walze type formula in this case. From now on, we always assume that $M$ carries a spin structure so that the spinor bundle and the Dirac operator are defined on $M$.

The following proposition is the key of the computation of lower-dimensional volumes of spin manifolds with boundary.

Proposition 6 (see [14]). The following identity holds:

(1) when $p_{1}+p_{2}=n$, then, $\operatorname{Vol}_{n}^{\left(p_{1}, p_{2}\right)} M=c_{0} \operatorname{Vol}_{M}$;

(2) when $p_{1}+p_{2} \equiv n \bmod 1, \quad \operatorname{Vol}_{n}^{\left(p_{1}, p_{2}\right)} M=\int_{\partial M} \Phi$.

Nextly, for 7-dimensional spin manifolds with boundary, we compute $\mathrm{Vol}_{7}^{(2,2)}$. By Proposition 6, for 7-dimensional compact manifolds with boundary, we have

$$
\widetilde{\text { Wres }}\left[\left(\pi^{+} D^{-2}\right)^{2}\right]=\int_{\partial M} \Phi \text {. }
$$

Recall the Dirac operator D of Definition 3. Write

$$
\begin{aligned}
D_{x}^{\alpha} & =(-\sqrt{-1})^{|\alpha|} \partial_{x}^{\alpha} ; \\
\sigma\left(D^{2}\right) & =p_{2}+p_{1}+p_{0} ; \quad \sigma\left(D^{-2}\right)=\sum_{j=1}^{\infty} q_{-j} .
\end{aligned}
$$

By the composition formula of pseudodifferential operators, then we have

$$
\begin{aligned}
1= & \sigma\left(D^{2} \cdot D^{-2}\right) \\
= & \sum_{\alpha} \frac{1}{\alpha !} \partial_{\xi}^{\alpha}\left[\sigma\left(D^{2}\right)\right] D_{x}^{\alpha}\left[\sigma\left(D^{-2}\right)\right] \\
= & \left(p_{2}+p_{1}+p_{0}\right)\left(q_{-2}+q_{-3}+q_{-4}+\cdots\right) \\
& +\sum_{j}\left(\partial_{\xi_{j}} p_{2}+\partial_{\xi_{j}} p_{1}+\partial_{\xi_{j}} p_{0}\right) \\
& \quad \times\left(D_{x_{j}} q_{-2}+D_{x_{j}} q_{-3}+D_{x_{j}} q_{-4}+\cdots\right)
\end{aligned}
$$

$$
\begin{aligned}
& +\sum_{i, j} \partial_{\xi_{i}} \partial_{\xi_{j}}\left(p_{2}+p_{1}+p_{0}\right) D_{x_{i}} D_{x_{j}} \\
& \times\left(q_{-2}+q_{-3}+q_{-4}+\cdots\right) \\
& =p_{2} q_{-2}+\left(p_{2} q_{-3}+p_{1} q_{-2}+\sum_{j} \partial_{\xi_{j}} p_{2} D_{x_{j}} q_{-2}\right) \\
& +\left(p_{2} q_{-4}+p_{1} q_{-3}+p_{0} q_{-2}+\sum_{j} \partial_{\xi_{j}} p_{2} D_{x_{j}} q_{-3}\right. \\
& \left.+\sum_{j} \partial_{\xi_{j}} p_{1} D_{x_{j}} q_{-2}+\sum_{i, j=1}^{n} \partial_{\xi_{i}} \partial_{\xi_{j}} p_{2} D_{x_{i}} D_{x_{j}} q_{-2}\right)+\cdots
\end{aligned}
$$

Thus, we get

$$
\begin{gathered}
q_{-2}=p_{2}^{-2} ; \\
q_{-3}=-p_{2}^{-1}\left[p_{1} q_{-2}-i \sum_{j=1}^{n} \partial_{\xi_{j}} p_{2} \partial_{x_{j}} q_{-2}\right] ; \\
q_{-4}=-p_{2}^{-1}\left[\begin{array}{c}
p_{0} q_{-2}+p_{1} q_{-3}-i \sum_{j=1}^{n} \partial_{\xi_{j}} p_{2} \partial_{x_{j}} q_{-3} \\
-i \sum_{j=1}^{n} \partial_{\xi_{j}} p_{1} \partial_{x_{j}} q_{-2} \\
-\frac{1}{2} \sum_{i, j=1}^{n} \partial_{\xi_{i}} \partial_{\xi_{j}} p_{2} \partial_{x_{i}} \partial_{x_{j}} q_{-2}
\end{array}\right] .
\end{gathered}
$$

Define $\Gamma^{k}=\sum_{i, j<n} \sum_{l<n} g^{i j} g^{l k}\left\langle\nabla_{\partial_{i}}^{L} \partial_{j}, \partial_{l}\right\rangle+\sum_{l<n} g^{l k}\left\langle\nabla_{\partial_{n}}^{L} \partial_{n}\right.$, $\left.\partial_{l}\right\rangle$ and $\sigma^{k}\left(x_{0}=(1 / 4) \sum_{s, t} \omega_{s, t}\left(\partial_{i}\right) c\left(\widetilde{e_{s}}\right) c\left(\widetilde{e_{t}}\right)\right.$. By Theorem 1 in [6] and Lemma 2.1 in [13], we have the following.

Lemma 7. Consider the symbol of the Dirac operator

$$
\begin{gathered}
\sigma_{-2}\left(D^{-2}\right)=|\xi|^{-2} ; \\
\sigma_{-3}\left(D^{-2}\right)=-\sqrt{-1}|\xi|^{-4} \xi_{k}\left(\Gamma^{k}-2 \sigma^{k}\right) \\
-\sqrt{-1}|\xi|^{-6} 2 \xi^{j} \xi_{\alpha} \xi_{\beta} \partial_{j} g^{\alpha \beta}
\end{gathered}
$$




$$
\begin{aligned}
\sigma_{-4}\left(D^{-2}\right)= & -|\xi|^{-6} \xi_{\mu} \xi_{\nu}\left(\Gamma^{\mu}-2 \sigma^{\mu}\right)\left(\Gamma^{\nu}-2 \sigma^{\nu}\right) \\
& +2|\xi|^{-8} \xi^{\mu} \xi_{\nu} \xi_{\alpha} \xi_{\beta}\left(\Gamma^{\nu}-2 \sigma^{\nu}\right) \partial_{\mu}^{x} g^{\alpha \beta} \\
& +|\xi|^{-4}\left(\partial^{x_{\mu}} \sigma_{\mu}+\sigma^{\mu} \sigma_{\mu}-\Gamma^{\mu} \sigma_{\mu}\right)-\frac{1}{4}|\xi|^{-4} s(x) \\
& -2|\xi|^{-6} \xi^{\mu} \xi_{\nu} \partial_{\mu}^{x}\left(\Gamma^{\nu}-2 \sigma^{\nu}\right) \\
& +12|\xi|^{-10} \xi^{\mu} \xi_{\nu} \xi_{\alpha} \xi_{\beta} \xi_{\gamma} \xi_{\delta} \partial_{\mu}^{x} g^{\alpha \beta} \partial_{\nu}^{x} g^{\gamma \delta} \\
& -4|\xi|^{-8} \xi^{\mu} \xi_{\alpha} \xi_{\gamma} \xi_{\delta} \partial_{\mu}^{x} g^{\nu \alpha} \partial_{\nu}^{x} g^{\gamma \delta} \\
& -4|\xi|^{-8} \xi^{\mu} \xi^{\nu} \xi_{\gamma} \xi_{\delta} \partial_{\mu \nu}^{x} g^{\gamma \delta} \\
& +|\xi|^{-6} \xi_{\alpha} \xi_{\beta}\left(\Gamma^{\mu}-2 \sigma^{\mu}\right) \partial_{\mu}^{x} g^{\alpha \beta} \\
& -|\xi|^{-6} \xi_{\alpha} \xi_{\beta} g^{\mu \nu} \partial_{\mu \nu}^{x} g^{\alpha \beta} \\
& +2|\xi|^{-8} \xi_{\alpha} \xi_{\beta} \xi_{\gamma} \xi_{\delta} g^{\mu \nu} \partial_{\mu}^{x} g^{\alpha \beta} \partial_{\nu}^{x} g^{\gamma \delta}
\end{aligned}
$$

where

$$
\begin{aligned}
\Gamma^{k}\left(x_{0}\right) & = \begin{cases}0, & \text { if } k<n ; \\
3 h^{\prime}(0) \xi_{n}, & \text { if } k=n,\end{cases} \\
\sigma^{k}\left(x_{0}\right) & = \begin{cases}\frac{1}{4} h^{\prime}(0) \sum_{k<n} \xi_{k} c\left(\widetilde{e_{k}}\right) c\left(\widetilde{e_{n}}\right), & \text { if } k<n ; \\
0, & \text { if } k=n .\end{cases}
\end{aligned}
$$

Since $\Phi$ is a global form on $\partial M$, so for any fixed point $x_{0} \in \partial M$, we can choose the normal coordinates $U$ of $x_{0}$ in $\partial M$ (not in $M$ ) and compute $\Phi\left(x_{0}\right)$ in the coordinates $\widetilde{U}=$ $U \times[0,1)$ and the metric $\left(1 / h\left(x_{n}\right)\right) g^{\partial M}+d x_{n}^{2}$. The dual metric of $g^{M}$ on $\widetilde{U}$ is $h\left(x_{n}\right) g^{\partial M}+d x_{n}^{2}$. Write $g_{i j}^{M}=g^{M}\left(\partial / \partial x_{i}, \partial / \partial x_{j}\right)$ and $g_{M}^{i j}=g^{M}\left(d x_{i}, d x_{j}\right)$; then,

$$
\begin{aligned}
& {\left[g_{i, j}^{M}\right]=\left[\begin{array}{cc}
\frac{1}{h\left(x_{n}\right)}\left[g_{i, j}^{\partial M}\right] & 0 \\
0 & 1
\end{array}\right] ;} \\
& {\left[g_{M}^{i, j}\right]=\left[\begin{array}{cc}
h\left(x_{n}\right)\left[g_{\partial M}^{i, j}\right] & 0 \\
0 & 1
\end{array}\right],} \\
& \partial_{x_{s}} g_{i j}^{\partial M}\left(x_{0}\right)=0, \quad 1 \leq i, j \leq n-1 ; \quad g_{i, j}^{M}\left(x_{0}\right)=\delta_{i j} .
\end{aligned}
$$

Let $\left\{E_{1}, \ldots, E_{n-1}\right\}$ be an orthonormal frame field in $U$ about $g^{\partial M}$ which is parallel along geodesics and $E_{i}=\left(\partial / \partial x_{i}\right)\left(x_{0}\right)$; then, $\left\{\widetilde{E_{1}}=\sqrt{h\left(x_{n}\right)} E_{1}, \ldots, \widetilde{E_{n-1}}=\right.$ $\left.\sqrt{h\left(x_{n}\right)} E_{n-1}, \widetilde{E_{n}}=d x_{n}\right\}$ is the orthonormal frame field in $\widetilde{U}$ about $g^{M}$. Locally, $S(T M) \mid \widetilde{U} \cong \widetilde{U} \times \wedge_{C}^{*}(n / 2)$. Let $\left\{f_{1}, \ldots, f_{n}\right\}$ be the orthonormal basis of $\wedge_{C}^{*}(n / 2)$. Take a spin frame field $\sigma: \widetilde{U} \rightarrow \operatorname{Spin}(M)$ such that $\pi \sigma=\left\{\widetilde{E_{1}}, \ldots, \widetilde{E_{n}}\right\}$, where $\pi: \operatorname{Spin}(M) \rightarrow O(M)$ is a double covering; then, $\left\{\left[\sigma, f_{i}\right], 1 \leq i \leq 6\right\}$ is an orthonormal frame of $\left.S(T M)\right|_{\widetilde{U}}$. In the following, since the global form $\Phi$ is independent of the choice of the local frame, we can compute $\operatorname{tr}_{S(T M)}$ in the frame $\left\{\left[\sigma, f_{i}\right], 1 \leq i \leq 6\right\}$. Let $\left\{\widehat{E}_{1}, \ldots, \widehat{E}_{n}\right\}$ be the canonical basis of $R^{n}$ and let $c\left(\widehat{E}_{i}\right) \in \operatorname{cl}_{C}(n) \cong \operatorname{Hom}\left(\wedge_{C}^{*}(n / 2), \wedge_{C}^{*}(n / 2)\right)$ be the Clifford action. By [13], then

$$
\begin{gathered}
c\left(\widetilde{E}_{i}\right)=\left[\left(\sigma, c\left(\widehat{E}_{i}\right)\right)\right] ; \\
c\left({\widetilde{E_{i}}}\right)\left[\left(\sigma, f_{i}\right)\right]=\left[\sigma,\left(c\left(\widehat{E}_{i}\right)\right) f_{i}\right] ; \\
\frac{\partial}{\partial x_{i}}=\left[\left(\sigma, \frac{\partial}{\partial x_{i}}\right)\right] ;
\end{gathered}
$$

then, we have $\left(\partial / \partial x_{i}\right) c\left(\widetilde{E_{i}}\right)=0$ in the above frame. By Lemma 2.2 in [13], we have the following.

Lemma 8. With the metric $g^{M}$ on $M$ near the boundary,

$$
\begin{aligned}
& \partial_{x_{j}}\left(|\xi|_{g^{M}}^{2}\right)\left(x_{0}\right)= \begin{cases}0, & \text { if } j<n ; \\
h^{\prime}(0)\left|\xi^{\prime}\right|_{g^{\partial M}}^{2}, & \text { if } j=n,\end{cases} \\
& \partial_{x_{j}}[c(\xi)]\left(x_{0}\right)= \begin{cases}0, & \text { if } j<n ; \\
\partial x_{n}\left(c\left(\xi^{\prime}\right)\right)\left(x_{0}\right), & \text { if } j=n,\end{cases}
\end{aligned}
$$

where $\xi=\xi^{\prime}+\xi_{n} d x_{n}$.

Then, the following lemma is introduced.

Lemma 9. The following identity holds:

$$
\partial_{x_{i}}\left[A_{\beta s}^{\alpha}\right]\left(x_{0}\right)= \begin{cases}R_{\beta i s \alpha}^{\partial_{M}}\left(x_{0}\right), & \text { if } i<n ; \\ 0, & \text { if } i=n .\end{cases}
$$

Proof. From Lemma 5.7 in [20], we have

$$
A_{\beta s}^{\alpha}=R_{\beta l s \alpha} x_{l}+O\left(|x|^{2}\right) .
$$

Then, we obtain $\partial_{x_{i}}\left[A_{\beta s}^{\alpha}\right]\left(x_{0}\right)=R_{\beta i s \alpha}^{\partial_{M}}\left(x_{0}\right)$. 
Lemma 10. Let $g^{M}$ be the metric on 7-dimensional spin manifolds $M$ near the boundary; then,

$$
\begin{gathered}
\left.\partial_{x_{\gamma}} \Gamma^{k}\left(x_{0}\right)\right|_{\left|\xi^{\prime}\right|=1}= \begin{cases}\frac{5}{6} \sum_{i<n} R_{i \gamma i k}^{\partial_{M}}\left(x_{0}\right), & \text { if } \gamma<n, k<n ; \\
0, & \text { if } \gamma<n, k=n ; \\
3 h^{\prime \prime}(0)-\frac{9}{2}\left(h^{\prime}(0)\right)^{2}, & \text { if } \gamma=n, k<n ;\end{cases} \\
\left.\partial_{x_{\gamma}} \sigma^{k}\left(x_{0}\right)\right|_{\left|\xi^{\prime}\right|=1} \\
= \begin{cases}\frac{1}{8} \sum_{s \neq t<n} R_{k \gamma s t}^{\partial_{M}}\left(x_{0}\right) c\left(\widetilde{E_{s}}\right) c\left(\widetilde{E_{t}}\right), & \text { if } \gamma<n, k<n ; \\
0, & \text { if } \gamma<n, k=n ; \\
\sum_{t<n}\left(\frac{3}{8}\left(h^{\prime}(0)\right)^{2}-\frac{1}{4} h^{\prime \prime}(0)\right) & \text { if } \gamma=n, k<n ; \\
\times c\left(\widetilde{E_{n}}\right) c\left(\widetilde{E_{t}}\right), & \text { if } \gamma=n, k=n . \\
\frac{1}{8} \sum_{t<n}\left(\left(h^{\prime}(0)\right)^{2}-h^{\prime \prime}(0)\right) & \left(\widetilde{E_{s}}\right) c\left(\widetilde{E_{t}}\right) .\end{cases}
\end{gathered}
$$

Proof. From Lemma 2.3 in [13], we have

$$
\Gamma^{k}=\sum_{i, j<n} \sum_{l<n} g^{i j} g^{l k}\left\langle\nabla_{\partial_{i}}^{L} \partial_{j}, \partial_{l}\right\rangle+\sum_{l<n} g^{l k}\left\langle\nabla_{\partial_{n}}^{L} \partial_{n}, \partial_{l}\right\rangle .
$$

Then,

$$
\begin{aligned}
\partial_{x_{\gamma}} \Gamma^{k}= & \sum_{i, j<n} \sum_{l<n} \partial_{x_{\gamma}}\left(g^{i j}\right) g^{l k}\left\langle\nabla_{\partial_{i}}^{L} \partial_{j}, \partial_{l}\right\rangle \\
& +\sum_{i, j<n} \sum_{l<n} g^{i j} \partial_{x_{\gamma}}\left(g^{l k}\right)\left\langle\nabla_{\partial_{i}}^{L} \partial_{j}, \partial_{l}\right\rangle \\
& +\sum_{i, j<n} \sum_{l<n} g^{i j} g^{l k} \partial_{x_{\nu}}\left(\left\langle\nabla_{\partial_{i}}^{L} \partial_{j}, \partial_{l}\right\rangle\right) \\
& +\sum_{l<n} \partial_{x_{\gamma}}\left(g^{l k}\right)\left\langle\nabla_{\partial_{n}}^{L} \partial_{n}, \partial_{l}\right\rangle \\
& +\sum_{l<n} g^{l k} \partial_{x_{\gamma}}\left(\left\langle\nabla_{\partial_{n}}^{L} \partial_{n}, \partial_{l}\right\rangle\right) .
\end{aligned}
$$

Let $H_{n n}=\left\langle\partial_{n}, \widetilde{E_{n}}\right\rangle=1, H_{n j}=\left\langle\partial_{n}, \widetilde{E_{j}}\right\rangle=0$, and $H_{i j}=$ $\left\langle\partial_{j}, \widetilde{E_{i}}\right\rangle_{M}=\left(1 / \sqrt{h\left(x_{n}\right)}\right)\left\langle\partial_{j}, E_{i}\right\rangle_{\partial_{M}}$. Then,

$$
\begin{aligned}
\left\langle\nabla_{\partial_{i}}^{L} \partial_{j}, \partial_{l}\right\rangle= & \left\langle\nabla_{\partial_{i}}^{L}\left(\left\langle\partial_{j}, \widetilde{E_{j_{1}}}\right\rangle \widetilde{E_{j_{1}}}\right),\left\langle\partial_{l}, \widetilde{E_{l_{1}}}\right\rangle \widetilde{E_{l_{1}}}\right\rangle \\
= & \partial_{i}\left(\left\langle\partial_{j}, \widetilde{E_{j_{1}}}\right\rangle\right)\left\langle\partial_{l}, \widetilde{E_{l_{1}}}\right\rangle\left\langle\widetilde{E_{j_{1}}}, \widetilde{E_{l_{1}}}\right\rangle \\
& +\left\langle\partial_{l}, \widetilde{E_{l_{1}}}\right\rangle\left\langle\partial_{j}, \widetilde{E_{j_{1}}}\right\rangle\left\langle\nabla \widetilde{\partial_{i}} L \widetilde{E_{j_{1}}}, \widetilde{E_{l_{1}}}\right\rangle \\
= & \partial_{i}\left(H_{j j_{1}}\right)\left\langle\partial_{l}, \widetilde{E_{l_{1}}}\right\rangle \delta_{j_{1}}^{l_{1}} \\
& +\left\langle\partial_{l}, \widetilde{E_{l_{1}}}\right\rangle\left\langle\partial_{j}, \widetilde{E_{j_{1}}}\right\rangle\left\langle\nabla_{\partial_{i}}^{L} \widetilde{E_{j_{1}}}, \widetilde{E_{l_{1}}}\right\rangle .
\end{aligned}
$$

When $i<n, j<n$,

$$
\begin{aligned}
\left\langle\nabla_{\partial_{i}}^{L} \partial_{j}, \partial_{l}\right\rangle\left(x_{0}\right)= & \partial_{i}\left(H_{j j_{1}}\right)\left\langle\partial_{l}, \widetilde{E_{l_{1}}}\right\rangle \delta_{j_{1}}^{l_{1}}\left(x_{0}\right) \\
& +\left\langle\partial_{l}, \widetilde{E_{l_{1}}}\right\rangle\left\langle\partial_{j}, \widetilde{E_{j_{1}}}\right\rangle\left\langle\nabla_{\partial_{i}}^{L} \widetilde{E_{j_{1}}}, \widetilde{E_{l_{1}}}\right\rangle\left(x_{0}\right) \\
= & \delta_{l_{1}}^{l} \delta_{j_{1}}^{j}\left\langle\nabla \nabla_{\partial_{i}}^{L} \widetilde{E_{j_{1}}}, \widetilde{E_{l_{1}}}\right\rangle\left(x_{0}\right) \\
= & \frac{\sqrt{h\left(x_{0}\right)}}{2} A_{i j}^{l}\left(x_{0}\right)=0, \\
& \left\langle\nabla_{\partial_{n}}^{L} \partial_{n}, \partial_{l}\right\rangle\left(x_{0}\right)=0 .
\end{aligned}
$$

Then

$$
\begin{aligned}
\partial_{x_{\gamma}} \Gamma^{k} & \left(x_{0}\right) \\
= & \sum_{i, l<n} \partial_{x_{\gamma}}\left(\left\langle\nabla_{\partial_{i}}^{L} \partial_{j}, \partial_{l}\right\rangle\right)\left(x_{0}\right)+\sum_{l<n} \partial_{x_{\gamma}}\left(\left\langle\nabla_{\partial_{n}}^{L} \partial_{n}, \partial_{l}\right\rangle\right)\left(x_{0}\right) \\
= & \partial_{\gamma} \partial_{i}\left(H_{j j_{1}}\right)\left\langle\partial_{l}, \widetilde{E_{l_{1}}}\right\rangle+\partial_{i}\left(H_{j j_{1}}\right) \partial_{\gamma}\left(\left\langle\partial_{l}, \widetilde{E_{l_{1}}}\right\rangle\right)\left(x_{0}\right) \\
& +\left(\partial_{\gamma}\left(\left\langle\partial_{l}, \widetilde{E_{l_{1}}}\right\rangle\right)\left\langle\partial_{j}, \widetilde{E_{j_{1}}}\right\rangle+\left\langle\partial_{l}, \widetilde{E_{l_{1}}}\right\rangle \partial_{\gamma}\left(\left\langle\partial_{j}, \widetilde{E_{j_{1}}}\right\rangle\right)\right) \\
& \times\left\langle\nabla_{\partial_{i}}^{L} \widetilde{E_{j_{1}}}, \widetilde{E_{l_{1}}}\right\rangle\left(x_{0}\right) \\
& +\left\langle\partial_{l}, \widetilde{E_{l_{1}}}\right\rangle\left\langle\partial_{j}, \widetilde{E_{j_{1}}}\right\rangle \partial_{\gamma}\left(\left\langle\nabla_{\partial_{i}}^{L} \widetilde{E_{j_{1}}}, \widetilde{E_{l_{1}}}\right\rangle\right)\left(x_{0}\right) \\
& +\partial_{\gamma} \partial_{i}\left(H_{n n_{1}}\right)\left\langle\partial_{l}, \widetilde{E_{l_{1}}}\right\rangle+\partial_{i}\left(H_{n n_{1}}\right) \partial_{\gamma}\left(\left\langle\partial_{l}, \widetilde{E_{l_{1}}}\right\rangle\right)\left(x_{0}\right) \\
& +\left(\partial_{\gamma}\left(\left\langle\partial_{l}, \widetilde{E_{l_{1}}}\right\rangle\right)\left\langle\partial_{n}, \widetilde{E_{n_{1}}}\right\rangle+\left\langle\partial_{l}, \widetilde{E_{l_{1}}}\right\rangle \partial_{\gamma}\left(\left\langle\partial_{n}, \widetilde{E_{n_{1}}}\right\rangle\right)\right) \\
& \times\left\langle\nabla_{\partial_{n}}^{L} \widetilde{E_{n_{1}}}, \widetilde{E_{l_{1}}}\right\rangle\left(x_{0}\right) \\
& +\left\langle\partial_{l}, \widetilde{E_{l_{1}}}\right\rangle\left\langle\partial_{n}, \widetilde{E_{n_{1}}}\right\rangle \partial_{\gamma}\left(\left\langle\nabla_{\partial_{n}}^{L} \widetilde{E_{n_{1}}}, \widetilde{E_{l_{1}}}\right\rangle\right)\left(x_{0}\right) \\
= & \partial_{\gamma} \partial_{i}\left(H_{j l}\right)\left(x_{0}\right)+\partial_{\gamma}\left(\left\langle\nabla_{\partial_{i}}^{L} \widetilde{E_{j}}, \widetilde{E_{l}}\right\rangle\right)\left(x_{0}\right) \\
= & -\frac{1}{3} \sum_{i l<n} R_{i \gamma l i}^{\partial_{M}}\left(x_{0}\right)+\partial_{\gamma}\left(\frac{\sqrt{h\left(x_{n}\right)}}{2} A_{i j}^{l}\right)\left(x_{0}\right) \\
= & \frac{5}{6} \sum_{i<n} R_{i j i k}^{\partial_{M}}\left(x_{0}\right) . \\
&
\end{aligned}
$$

Similarly, when $\gamma<n, k=n$, or $\gamma=n, k<n, \partial_{x_{\gamma}} \Gamma^{k}\left(x_{0}\right)=0$. When $\gamma=k=n, \partial_{x_{\gamma}} \Gamma^{k}\left(x_{0}\right)=3 h^{\prime \prime}(0)-(9 / 2)\left(h^{\prime}(0)\right)^{2}$.

On the other hand, from definitions (10) and (11), then

$$
\sigma^{k}\left(x_{0}\right)=\frac{1}{4} \sum_{s, t} \omega_{s, t}\left(\partial_{i}\right) c\left(\widetilde{E_{s}}\right) c\left(\widetilde{E_{t}}\right) .
$$


When $\gamma<n, k<n$,

$$
\begin{aligned}
\partial_{x_{\gamma}} \sigma^{k}\left(x_{0}\right) & \\
= & \partial_{x_{\nu}}\left(\frac{1}{4} \sum_{s, t} \omega_{s, t}\left(\partial_{k}\right) c\left(\widetilde{E_{s}}\right) c\left(\widetilde{E_{t}}\right)\right)\left(x_{0}\right) \\
= & \frac{1}{4} \partial_{x_{\nu}}\left(\sum_{s, t} \omega_{s, t}\left(\partial_{k}\right)\right)\left(x_{0}\right) c\left(\widetilde{E_{s}}\right) c\left(\widetilde{E_{t}}\right) \\
= & \frac{1}{4}\left(\sum_{s, t<n} \partial_{x_{\gamma}} \omega_{s, t}\left(\partial_{k}\right)+\sum_{s=n, t<n} \partial_{x_{\gamma}} \omega_{s, t}\left(\partial_{k}\right)\right. \\
& \left.+\sum_{s<n, t=n} \partial_{x_{\gamma}} \omega_{s, t}\left(\partial_{k}\right)\right)\left(x_{0}\right) c\left(\widetilde{E_{s}}\right) c\left(\widetilde{E_{t}}\right) \\
= & \frac{1}{4}\left(\sum_{s, t<n} \partial_{x_{\gamma}}\left\langle\nabla_{\partial_{x_{k}}}^{L} \widetilde{E_{s}}, \widetilde{E_{t}}\right\rangle c\left(\widetilde{E_{s}}\right) c\left(\widetilde{E_{t}}\right)\right. \\
& +\sum_{s=n, t<n} \partial_{x_{\gamma}}\left\langle\nabla_{\partial_{x_{k}}}^{L} \widetilde{E_{s}}, \widetilde{E_{t}}\right\rangle c\left(\widetilde{E_{s}}\right) c\left(\widetilde{E_{t}}\right) \\
& \left.+\sum_{s<n, t=n} \partial_{x_{\gamma}}\left\langle\nabla_{\partial_{x_{k}}}^{L} \widetilde{E_{s}}, \widetilde{E_{t}}\right\rangle c\left(\widetilde{E_{s}}\right) c\left(\widetilde{E_{t}}\right)\right)\left(x_{0}\right) \\
= & \frac{1}{4} \sum_{s, t<n} \partial_{\gamma}\left(\frac{\sqrt{h\left(x_{n}\right)}}{2} \sum_{s \neq t<n} R_{k s}^{\partial_{M}}\left(x_{0}\right) c\left(\widetilde{E_{s}}\right) c\left(\widetilde{E_{t}}\right) .\right. \\
& \left(x_{0}\right)
\end{aligned}
$$

Similarly, when $\gamma=n, k<n, \partial_{x_{\gamma}} \sigma^{k}\left(x_{0}\right)=$ $\sum_{t<n}\left((3 / 8)\left(h^{\prime}(0)\right)^{2}-(1 / 4) h^{\prime \prime}(0)\right) c\left(\widetilde{E_{n}}\right) c\left(\widetilde{E_{t}}\right)$. When $\gamma<n$, $k=n, \partial_{x_{\gamma}} \sigma^{k}\left(x_{0}\right)=0$. When $\gamma=k=n, \partial_{x_{\gamma}} \sigma^{k}\left(x_{0}\right)=$ $(1 / 8) \sum_{t<n}\left(\left(h^{\prime}(0)\right)^{2}-h^{\prime \prime}(0)\right) c\left(\widetilde{E_{s}}\right) c\left(\widetilde{E_{t}}\right)$.

Lemma 11. When $\gamma<n$,

$$
\begin{aligned}
\left.\partial_{x_{\nu}}\left(\sigma_{-3}\left(D^{-2}\right)\right)\left(x_{0}\right)\right|_{\left|\xi^{\prime}\right|=1} \\
=-\frac{5 i}{6} \frac{\xi_{k}}{\left(1+\xi_{n}^{2}\right)^{2}} \sum_{i<n} R_{i \gamma i k}^{\partial_{M}}\left(x_{0}\right) \\
+\frac{i}{4} \frac{\xi_{k}}{\left(1+\xi_{n}^{2}\right)^{2}} \sum_{s \neq t<n} R_{k \gamma s t}^{\partial_{M}}\left(x_{0}\right) c\left(\widetilde{E_{s}}\right) c\left(\widetilde{E_{t}}\right) \\
+\frac{2 i}{3} \frac{1}{\left(1+\xi_{n}^{2}\right)^{3}} \sum_{\alpha, \beta<n}\left(R_{i \alpha j \beta}^{\partial_{M}}\left(x_{0}\right)+R_{i \beta j \alpha}^{\partial_{M}}\left(x_{0}\right)\right) \xi_{j} \xi_{\alpha} \xi_{\beta} .
\end{aligned}
$$

When $\gamma=n$,

$$
\begin{aligned}
\left.\partial_{x_{n}}\left(\sigma_{-3}\left(D^{-2}\right)\right)\left(x_{0}\right)\right|_{\left|\xi^{\prime}\right|=1} \\
=\frac{2 i h^{\prime}(0)}{\left(1+\xi_{n}^{2}\right)^{3}}\left(-\frac{1}{2} h^{\prime}(0) \sum_{k<n} \xi_{k} c\left(\widetilde{e_{k}}\right) c\left(\widetilde{e_{n}}\right)+3 h^{\prime}(0) \xi_{n}\right) \\
\quad-\frac{i}{\left(1+\xi_{n}^{2}\right)^{2}} \\
\quad \times\left(\xi_{n}\left(3 h^{\prime \prime}(0)-\frac{9}{2}\left(h^{\prime}(0)\right)^{2}\right)\right. \\
\quad-2 \xi_{k}\left(\frac{3}{8}\left(h^{\prime}(0)\right)^{2}-\frac{1}{4} h^{\prime \prime}(0)\right) \sum_{t<n} c\left(\widetilde{E_{n}}\right) c\left(\widetilde{E_{t}}\right) \\
\left.\quad-\frac{1}{4} \xi_{n}\left(\left(h^{\prime}(0)\right)^{2}-h^{\prime \prime}(0)\right) \sum_{s \neq t<n} c\left(\widetilde{E_{s}}\right) c\left(\widetilde{E_{t}}\right)\right) .
\end{aligned}
$$

Proof. When $\gamma<n$, from Lemmas 7 and 8 and $\partial_{x_{y}}\left(c\left(d x_{j}\right)\right)\left(x_{0}\right)=0$, we get

$$
\begin{aligned}
\partial_{x_{\nu}} & \left(\sigma_{-3}\left(D^{-2}\right)\right) \\
= & \partial_{x_{\gamma}}\left(-\sqrt{-1}|\xi|^{-4} \xi_{k}\left(\Gamma^{k}-2 \sigma^{k}\right)-\sqrt{-1}|\xi|^{-6} 2 \xi^{j} \xi_{\alpha} \xi_{\beta} \partial_{j} g^{\alpha \beta}\right) \\
= & -\sqrt{-1}|\xi|^{-4} \xi_{k} \partial_{x_{\nu}}\left(\Gamma^{k}-2 \sigma^{k}\right) \\
& -\sqrt{-1}|\xi|^{-6} 2 \xi^{j} \xi_{\alpha} \xi_{\beta} \partial_{x_{\gamma}} \partial_{j} g^{\alpha \beta} \\
= & -\sqrt{-1}|\xi|^{-4}\left(\xi_{k} \partial_{x_{\gamma}}\left(\Gamma^{k}-2 \sigma^{k}\right)+\xi_{n} \partial_{x_{\gamma}}\left(\Gamma^{n}-2 \sigma^{n}\right)\right) \\
& -\sqrt{-1}|\xi|^{-6} 2 \xi^{j} \xi_{\alpha} \xi_{\beta} \partial_{x_{\gamma}} \partial_{j} g^{\alpha \beta} .
\end{aligned}
$$

Substituting Lemma 10 into (47), conclusion (45) then follows easily. Similarly, we can obtain (45).

Next, we can compute $\Phi$ (see formula (23) for definition of $\Phi)$. Since the sum is taken over $-r-\ell+1+k+j+|\alpha|=7$, $r, \ell \leq-2$, then we have that $\int_{\partial_{M}} \Phi$ is the sum of the following fifteen cases.

Case 1. Consider $r=-2, \ell=-2, k=0, j=1$, and $|\alpha|=1$.

From (23), we have

Case 1

$$
\begin{gathered}
=\frac{i}{2} \int_{\left|\xi^{\prime}\right|=1} \int_{-\infty}^{+\infty} \sum_{|\alpha|=1} \operatorname{trace}\left[\partial_{x_{n}} \partial_{\xi^{\prime}}^{\alpha} \pi_{\xi_{n}}^{+} \sigma_{-2}\left(D^{-2}\right)\right. \\
\left.\times \partial_{x^{\prime}}^{\alpha} \partial_{\xi_{n}}^{2} \sigma_{-2}\left(D^{-2}\right)\right] \\
\times\left(x_{0}\right) \mathrm{d} \xi_{n} \sigma\left(\xi^{\prime}\right) \mathrm{d} x^{\prime} .
\end{gathered}
$$

By Lemma 8 , for $i<n$, we have

$$
\partial_{x_{i}} \sigma_{-2}\left(D^{-2}\right)\left(x_{0}\right)=\partial_{x_{i}}\left(|\xi|^{-2}\right)\left(x_{0}\right)=0 .
$$

So Case 1 vanishes. 
Case 2. Consider $r=-2, \ell=-2, k=0, j=2$, and $|\alpha|=0$. From (23), we have

Case 2

$$
\begin{gathered}
=\frac{i}{6} \int_{\left|\xi^{\prime}\right|=1} \int_{-\infty}^{+\infty} \sum_{j=2} \operatorname{trace}\left[\partial_{x_{n}}^{2} \pi_{\xi_{n}}^{+} \sigma_{-2}\left(D^{-2}\right)\right. \\
\left.\times \partial_{\xi_{n}}^{3} \sigma_{-2}\left(D^{-2}\right)\right] \\
\times\left(x_{0}\right) \mathrm{d} \xi_{n} \sigma\left(\xi^{\prime}\right) \mathrm{d} x^{\prime} .
\end{gathered}
$$

By Lemma 7, a simple computation shows

$$
\begin{aligned}
\left.\partial_{\xi_{n}}^{3} \sigma_{-2}\left(D^{-2}\right)\left(x_{0}\right)\right|_{\left|\xi^{\prime}\right|=1} & =\partial_{\xi_{n}}^{3}\left(\frac{1}{1+\xi_{n}^{2}}\right)=\frac{24 \xi_{n}-24 \xi_{n}^{3}}{\left(1+\xi_{n}^{2}\right)^{4}} \\
\partial_{x_{n}}^{2} \sigma_{-2}\left(D^{-2}\right)\left(x_{0}\right)= & \frac{2}{|\xi|^{6}} \partial_{x_{n}}\left(|\xi|^{2}\right) \partial_{x_{n}}\left(|\xi|^{2}\right)\left(x_{0}\right) \\
& -\frac{\partial_{x_{n}}^{2}\left(|\xi|^{2}\right)}{|\xi|^{6}}\left(x_{0}\right) \\
= & \frac{2\left(h^{\prime}(0)\right)^{2}}{\left(1+\xi_{n}^{2}\right)^{3}}-\frac{h^{\prime \prime}(0)}{\left(1+\xi_{n}^{2}\right)^{2}}
\end{aligned}
$$

By (18) and the Cauchy integral formula, then

$$
\begin{aligned}
\pi_{\xi_{n}}^{+} & {\left[\frac{c(\xi)}{\left(1+\xi_{n}^{2}\right)^{2}}\right] } \\
= & \pi_{\xi_{n}}^{+}\left[\frac{c\left(\xi^{\prime}\right)+\xi_{n} c\left(d x_{n}\right)}{\left(1+\xi_{n}^{2}\right)^{2}}\right] \\
= & \frac{1}{2 \pi i} \lim _{u \rightarrow 0^{-}} \int_{\Gamma^{+}}\left(\left(\frac{c\left(\xi^{\prime}\right)+\eta_{n} c\left(d x_{n}\right)}{\left(\eta_{n}+i\right)^{2}\left(\xi_{n}+i u-\eta_{n}\right)}\right)\right. \\
= & \left.\times \frac{\left.c\left(\eta_{n}-i\right)^{-2}\right) \mathrm{d} \eta_{n}}{\left(\eta_{n}+i\right)^{2}\left(\xi_{n}-\eta_{n}\right)}\right]\left.^{(1)}\right|_{\eta_{n}=i} \\
= & -\frac{i c\left(\xi^{\prime}\right)}{4\left(\xi_{n}-i\right)}-\frac{c\left(\xi^{\prime}\right)+i c\left(d x_{n}\right)}{4\left(\xi_{n}-i\right)^{2}} .
\end{aligned}
$$

Similarly, we obtain

$$
\begin{aligned}
& \pi_{\xi_{n}}^{+}\left[\frac{1}{\left(1+\xi_{n}^{2}\right)^{2}}\right]=\frac{-2-i \xi_{n}}{4\left(\xi_{n}-i\right)^{2}}, \\
& \pi_{\xi_{n}}^{+}\left[\frac{1}{\left(1+\xi_{n}^{2}\right)^{3}}\right]=\frac{-3 i \xi_{n}^{2}-9 \xi_{n}+8 i}{16\left(\xi_{n}-i\right)^{3}} .
\end{aligned}
$$

From (51) and (53), we get

$$
\begin{aligned}
\partial_{x_{n}}^{2} & \left.\pi_{\xi_{n}}^{+} \sigma_{-2}\left(D^{-2}\right)\left(x_{0}\right)\right|_{\left|\xi^{\prime}\right|=1} \\
& =\frac{-3 i \xi_{n}^{2}-9 \xi_{n}+8 i}{8\left(\xi_{n}-i\right)^{3}}\left(h^{\prime}(0)\right)^{2}+\frac{2+i \xi_{n}}{4\left(\xi_{n}-i\right)^{2}} h^{\prime \prime}(0) .
\end{aligned}
$$

Note that $\operatorname{tr}[\mathrm{id}]=8$; then, from (50), (54), and direct computations, we obtain

$$
\begin{aligned}
\operatorname{trace} & {\left.\left[\partial_{x_{n}}^{2} \pi_{\xi_{n}}^{+} \sigma_{-2}\left(D^{-2}\right) \partial_{\xi_{n}}^{3} \sigma_{-2}\left(D^{-2}\right)\right]\left(x_{0}\right)\right|_{\left|\xi^{\prime}\right|=1} } \\
= & \left(h^{\prime}(0)\right)^{2} \frac{\left(-3 i \xi_{n}^{2}-9 \xi_{n}+8 i\right)\left(24 \xi_{n}-24 \xi_{n}^{3}\right)}{\left(\xi_{n}-i\right)^{3}\left(1+\xi_{n}^{2}\right)^{4}} \\
+ & h^{\prime \prime}(0) \frac{\left(4+2 i \xi_{n}\right)\left(24 \xi_{n}-24 \xi_{n}^{3}\right)}{\left(\xi_{n}-i\right)^{2}\left(1+\xi_{n}^{2}\right)^{4}} .
\end{aligned}
$$

Therefore,

Case 2

$$
\begin{aligned}
& =\frac{i}{6}\left(h^{\prime}(0)\right)^{2} \\
& \quad \times \int_{\left|\xi^{\prime}\right|=1} \int_{-\infty}^{+\infty}\left(\left(\left(-3 i \xi_{n}^{2}-9 \xi_{n}+8 i\right)\left(24 \xi_{n}-24 \xi_{n}^{3}\right)\right)\right. \\
& \left.\quad \times\left(\left(\xi_{n}-i\right)^{3}\left(1+\xi_{n}^{2}\right)^{4}\right)^{-1}\right) \mathrm{d} \xi_{n} \sigma\left(\xi^{\prime}\right) \mathrm{d} x^{\prime} \\
& +\frac{i}{6} h^{\prime \prime}(0) \quad \int_{\left|\xi^{\prime}\right|=1} \int_{-\infty}^{+\infty} \frac{\left(4+2 i \xi_{n}\right)\left(24 \xi_{n}-24 \xi_{n}^{3}\right)}{\left(\xi_{n}-i\right)^{2}\left(1+\xi_{n}^{2}\right)^{4}} \mathrm{~d} \xi_{n} \sigma\left(\xi^{\prime}\right) \mathrm{d} x^{\prime} \\
& =\frac{i}{6}\left(h^{\prime}(0)\right)^{2} \Omega_{5} \\
& \quad \times \int_{\Gamma^{+}} \frac{\left(-3 i \xi_{n}^{2}-9 \xi_{n}+8 i\right)\left(24 \xi_{n}-24 \xi_{n}^{3}\right)}{\left(\xi_{n}-i\right)^{7}\left(\xi_{n}+i\right)^{4}} \mathrm{~d} \xi_{n} \mathrm{~d} x^{\prime} \\
& +\frac{i}{6} h^{\prime \prime}(0) \Omega_{5} \int_{\Gamma^{+}} \frac{\left(4+2 i \xi_{n}\right)\left(24 \xi_{n}-24 \xi_{n}^{3}\right)}{\left(\xi_{n}-i\right)^{6}\left(\xi_{n}^{2}+i\right)^{4}} \mathrm{~d} \xi_{n} \mathrm{~d} x^{\prime} \\
& =\frac{i}{6}\left(h^{\prime}(0)\right)^{2} \frac{2 \pi i}{6 !} \\
& \quad \times\left[\left.\frac{\left(-3 i \xi_{n}^{2}-9 \xi_{n}+8 i\right)\left(24 \xi_{n}-24 \xi_{n}^{3}\right)}{\left(\xi_{n}+i\right)^{4}}\right|_{\xi_{n}=i} ^{(6)} \Omega_{5} \mathrm{~d} x^{\prime}\right. \\
& +\frac{i}{6} h^{\prime \prime}(0) \frac{2 \pi i}{5 !}
\end{aligned}
$$




$$
\begin{aligned}
& \times\left.\left[\frac{\left(4+2 i \xi_{n}\right)\left(24 \xi_{n}-24 \xi_{n}^{3}\right)}{\left(\xi_{n}^{2}+i\right)^{4}}\right]^{(5)}\right|_{\xi_{n}=i} \Omega_{5} \mathrm{~d} x^{\prime} \\
= & \left(\frac{7}{8}\left(h^{\prime}(0)\right)^{2}-\frac{3}{8} h^{\prime \prime}(0)\right) \pi \Omega_{5} \mathrm{~d} x^{\prime},
\end{aligned}
$$

where $\Omega_{5}$ is the canonical volume of $S^{5}$.

Case 3. Consider $r=-2, \ell=-2, k=0, j=0$, and $|\alpha|=2$.

From (23), we have

Case 3

$$
\begin{aligned}
=\frac{i}{2} \int_{\left|\xi^{\prime}\right|=1} \int_{-\infty}^{+\infty} \sum_{|\alpha|=2} \operatorname{trace}\left[\partial_{\xi^{\prime}}^{\alpha} \pi_{\xi_{n}}^{+} \sigma_{-2}\right. \\
\left.\times\left(D^{-2}\right) \partial_{x^{\prime}}^{\alpha} \partial_{\xi_{n}} \sigma_{-2}\left(D^{-2}\right)\right] \\
\times\left(x_{0}\right) \mathrm{d} \xi_{n} \sigma\left(\xi^{\prime}\right) \mathrm{d} x^{\prime} .
\end{aligned}
$$

By Lemma 7, a simple computation shows

$$
\begin{aligned}
\left.\partial_{\xi^{\prime}}^{\alpha} \sigma_{-2}\left(D^{-2}\right)\left(x_{0}\right)\right|_{\left|\xi^{\prime}\right|=1}= & \left.\partial_{\xi_{j}} \partial_{\xi_{i}} \sigma_{-2}\left(D^{-2}\right)\left(x_{0}\right)\right|_{\left|\xi^{\prime}\right|=1} \\
= & \frac{-1}{\left(1+\xi_{n}^{2}\right)^{2}} \partial_{\xi_{i}} \partial_{\xi_{j}}\left(|\xi|^{2}\right)\left(x_{0}\right) \\
& +\frac{2}{\left(1+\xi_{n}^{2}\right)^{3}} \partial_{\xi_{j}}\left(|\xi|^{2}\right) \partial_{\xi_{i}}\left(|\xi|^{2}\right)\left(x_{0}\right) \\
= & \frac{-2 \delta_{i}^{j}}{\left(1+\xi_{n}^{2}\right)^{2}}+\frac{8}{\left(1+\xi_{n}^{2}\right)^{3}} \xi_{i} \xi_{j} .
\end{aligned}
$$

By (53) and (58), we obtain

$$
\begin{aligned}
\left.\pi_{\xi_{n}}^{+} \partial_{\xi^{\prime}}^{\alpha} \sigma_{-2}\left(D^{-2}\right)\left(x_{0}\right)\right|_{\left|\xi^{\prime}\right|=1}= & \frac{\left(2+i \xi_{n}\right) \delta_{i}^{j}}{2\left(\xi_{n}-i\right)^{2}} \\
& +\frac{-3 i \xi_{n}^{2}-9 \xi_{n}+8 i}{2\left(\xi_{n}-i\right)^{3}} \xi_{i} \xi_{j} .
\end{aligned}
$$

On the other hand, by Lemmas 7 and 8 , we obtain

$$
\begin{aligned}
\left.\partial_{x^{\prime}}^{\alpha} \sigma_{-2}\left(D^{-2}\right)\left(x_{0}\right)\right|_{\left|\xi^{\prime}\right|=1} & \\
= & \frac{-1}{\left(1+\xi_{n}^{2}\right)^{2}} \partial_{x_{i}} \partial_{x_{j}}\left(|\xi|^{2}\right)\left(x_{0}\right) \\
& +\frac{2}{\left(1+\xi_{n}^{2}\right)^{3}} \partial_{x_{j}}\left(|\xi|^{2}\right) \partial_{x_{i}}\left(|\xi|^{2}\right)\left(x_{0}\right) \\
= & \frac{1}{3\left(1+\xi_{n}^{2}\right)^{2}} \sum_{\alpha, \beta<n}\left(R_{i \alpha j \beta}^{\partial_{M}}\left(x_{0}\right)+R_{i \beta j \alpha}^{\partial_{M}}\left(x_{0}\right)\right) \xi_{\alpha} \xi_{\beta} \\
& +\frac{2\left(h^{\prime}(0)\right)^{2}}{\left(1+\xi_{n}^{2}\right)^{3}} .
\end{aligned}
$$

Hence, in this case,

$$
\begin{aligned}
\left.\partial_{x^{\prime}}^{\alpha} \partial_{\xi_{n}} \sigma_{-2}\left(D^{-2}\right)\left(x_{0}\right)\right|_{\left|\xi^{\prime}\right|=1} \\
=\frac{-4 \xi_{n}}{3\left(1+\xi_{n}^{2}\right)^{3}} \sum_{\alpha, \beta<n}\left(R_{i \alpha j \beta}^{\partial_{M}}\left(x_{0}\right)+R_{i \beta j \alpha}^{\partial_{M}}\left(x_{0}\right)\right) \xi_{\alpha} \xi_{\beta} \\
\quad+\frac{-12 \xi_{n}\left(h^{\prime}(0)\right)^{2}}{\left(1+\xi_{n}^{2}\right)^{4}} .
\end{aligned}
$$

From (59), (61), and direct computations, we obtain

$$
\begin{aligned}
\operatorname{trace} & {\left[\partial_{\xi^{\prime}}^{\alpha} \pi_{\xi_{n}}^{+} \sigma_{-2}\left(D^{-2}\right) \partial_{x^{\prime}}^{\alpha} \partial_{\xi_{n}} \sigma_{-2}\left(D^{-2}\right)\right]\left(x_{0}\right) } \\
= & \frac{-4 \xi_{n}-2 i \xi_{n}^{2}}{3\left(\xi_{n}-i\right)^{2}\left(1+\xi_{n}^{2}\right)^{3}} \sum_{\alpha, \beta<n}\left(R_{i \alpha j \beta}^{\partial_{M}}\left(x_{0}\right)+R_{i \beta j \alpha}^{\partial_{M}}\left(x_{0}\right)\right) \xi_{\alpha} \xi_{\beta} \\
& +\frac{-16 i \xi_{n}+18 \xi_{n}^{2}+6 i \xi_{n}^{3}}{3\left(\xi_{n}-i\right)^{3}\left(1+\xi_{n}^{2}\right)^{3}} \\
& \times \sum_{\alpha, \beta<n}\left(R_{i \alpha j \beta}^{\partial_{M}}\left(x_{0}\right)+R_{i \beta j \alpha}^{\partial_{M}}\left(x_{0}\right)\right) \xi_{i} \xi_{j} \xi_{\alpha} \xi_{\beta} \\
& +\left(h^{\prime}(0)\right)^{2} \frac{-12 \xi_{n}-6 i \xi_{n}^{2}}{\left(\xi_{n}-i\right)^{2}\left(1+\xi_{n}^{2}\right)^{4}} \\
& +\left(h^{\prime}(0)\right)^{2-48 i \xi_{n}+54 \xi_{n}^{2}+18 i \xi_{n}^{3}}
\end{aligned}
$$

Similar to (16) in [6], we have

$$
\int \xi^{\mu} \xi^{\nu}=\frac{1}{6}\left[{ }^{\mu \nu}\right], \quad \int \xi^{\mu} \xi^{\nu} \xi^{\alpha} \xi^{\beta}=c_{0}\left[{ }^{\mu \nu \alpha \beta}\right]
$$

where $\left[{ }^{\mu \nu \alpha \beta}\right]$ stands for the sum of products of $g^{\alpha \beta}$ determined by all "pairings" of $\mu \nu \alpha \beta$ and $c_{0}$ is a constant. Using the integration over $S^{5}$ and the shorthand $\int=\left(1 / \pi^{3}\right) \int_{S^{5}} \mathrm{~d}^{5} \nu$, we obtain $\Omega_{5}=\pi^{3}$. Let $s_{\partial_{M}}$ be the scalar curvature $\partial_{M}$; then,

$$
\begin{aligned}
& \sum_{i, \alpha, j, \beta<n} R_{i \alpha j \beta}^{\partial_{M}}\left(x_{0}\right) \int_{\left|\xi^{\prime}\right|=1} \xi_{\alpha} \xi_{\beta} \xi_{i} \xi_{j} \sigma\left(\xi^{\prime}\right)=c \pi^{3} \\
& \sum_{i, \alpha, j, \beta<n} R_{i \alpha j \beta}^{\partial_{M}}\left(x_{0}\right)\left(\delta_{\alpha}^{\beta} \delta_{i}^{j}+\delta_{\alpha}^{i} \delta_{\beta}^{j}+\delta_{\alpha}^{j} \delta_{\beta}^{i}\right)=0,
\end{aligned}
$$


where $c$ is a constant. Therefore,

Case 3

$$
\begin{gathered}
=\frac{i}{2} \Omega_{5}\left(s_{\partial_{M}} \int_{-\infty}^{+\infty} \frac{-4 \xi_{n}-2 i \xi_{n}^{2}}{9\left(\xi_{n}-i\right)^{2}\left(1+\xi_{n}^{2}\right)^{3}} \mathrm{~d} \xi_{n}\right. \\
\left.+\left(h^{\prime}(0)\right)^{2} \int_{-\infty}^{+\infty} \frac{4 i \xi_{n}-9 \xi_{n}^{2}-3 i \xi_{n}^{3}}{\left(\xi_{n}-i\right)^{3}\left(1+\xi_{n}^{2}\right)^{4}} \mathrm{~d} \xi_{n}\right) \mathrm{d} x^{\prime} \\
\left.=\frac{i}{2} \Omega_{5}\left(s_{\partial_{M}} \frac{2 \pi i}{4 !}\left[\frac{-4 \xi_{n}-2 i \xi_{n}^{2}}{9\left(\xi_{n}+i\right)^{3}}\right]_{\xi_{n}=i}^{(4)}\right]_{\xi_{n}=i}\right) \mathrm{d} x^{\prime} \\
+\left(h^{\prime}(0)\right)^{2} \frac{2 \pi i}{6 !}\left[\left.\left.\frac{4 i \xi_{n}-9 \xi_{n}^{2}-3 i \xi_{n}^{3}}{\left(\xi_{n}+i\right)^{4}}\right|^{(6)}\right|^{2}\right]^{2} \Omega_{5} \mathrm{~d} x^{\prime},
\end{gathered}
$$

where $\sum_{t, l<n} R_{t l t l}^{\partial_{M}}\left(x_{0}\right)$ is the scalar curvature $s_{\partial_{M}}$.

Case 4. Consider $r=-2, \ell=-2, k=1, j=1$, and $|\alpha|=0$.

From (23) and the Leibniz rule, we obtain

Case 4

$$
\begin{aligned}
=\frac{i}{6} \int_{\left|\xi^{\prime}\right|=1} \int_{-\infty}^{+\infty} \operatorname{trace} & {\left[\partial_{x_{n}} \partial_{\xi_{n}} \pi_{\xi_{n}}^{+} \sigma_{-2}\right.} \\
& \left.\times\left(D^{-2}\right) \partial_{\xi_{n}}^{2} \partial_{x_{n}} \sigma_{-2}\left(D^{-2}\right)\right] \\
& \times\left(x_{0}\right) \mathrm{d} \xi_{n} \sigma\left(\xi^{\prime}\right) \mathrm{d} x^{\prime} .
\end{aligned}
$$

By (54), we obtain

$$
\left.\partial_{x_{n}} \partial_{\xi_{n}} \pi_{\xi_{n}}^{+} \sigma_{-2}\left(D^{-2}\right)\left(x_{0}\right)\right|_{\left|\xi^{\prime}\right|=1}=h^{\prime}(0) \frac{-3-i \xi_{n}}{4\left(\xi_{n}-i\right)^{3}} .
$$

From (50) and (51), we obtain

$$
\left.\partial_{\xi_{n}}^{2} \partial_{x_{n}} \sigma_{-2}\left(D^{-2}\right)\left(x_{0}\right)\right|_{\left|\xi^{\prime}\right|=1}=h^{\prime}(0) \frac{4-20 \xi_{n}^{2}}{\left(1+\xi_{n}^{2}\right)^{4}}
$$

Therefore,

Case 4

$$
\begin{aligned}
& =\frac{i}{6}\left(h^{\prime}(0)\right)^{2} \\
& \quad \times \int_{\left|\xi^{\prime}\right|=1} \int_{-\infty}^{+\infty} \frac{-24-8 i \xi_{n}+120 \xi_{n}^{2}+40 i \xi_{n}^{3}}{\left(\xi_{n}-i\right)^{3}\left(1+\xi_{n}^{2}\right)^{4}} \mathrm{~d} \xi_{n} \sigma\left(\xi^{\prime}\right) \mathrm{d} x^{\prime}
\end{aligned}
$$

$$
\begin{aligned}
= & \frac{i}{6}\left(h^{\prime}(0)\right)^{2} \frac{2 \pi i}{6 !} \\
& \times\left.\left[\frac{-24-8 i \xi_{n}+120 \xi_{n}^{2}+40 i \xi_{n}^{3}}{\left(\xi_{n}+i\right)^{4}}\right]_{\xi_{n}=i}^{(6)}\right|_{5} \mathrm{~d} x^{\prime} \\
= & -\frac{5}{8}\left(h^{\prime}(0)\right)^{2} \pi \Omega_{5} \mathrm{~d} x^{\prime} .
\end{aligned}
$$

Case 5. Consider $r=-2, \ell=-2, k=1, j=0$, and $|\alpha|=1$.

$$
\text { From (23), we have }
$$

Case 5

$$
\begin{gathered}
=\frac{i}{2} \int_{\left|\xi^{\prime}\right|=1} \int_{-\infty}^{+\infty} \sum_{|\alpha|=1} \operatorname{trace}\left[\partial_{\xi^{\prime}}^{\alpha} \partial_{\xi_{n}} \pi_{\xi_{n}}^{+} \sigma_{-2}\right. \\
\left.\quad \times\left(D^{-2}\right) \partial_{x^{\prime}}^{\alpha} \partial_{\xi_{n}} \partial_{x_{n}} \sigma_{-2}\left(D^{-2}\right)\right] \\
\times\left(x_{0}\right) \mathrm{d} \xi_{n} \sigma\left(\xi^{\prime}\right) \mathrm{d} x^{\prime}
\end{gathered}
$$

From Lemmas 7 and 8, for $i<n$, we obtain

$$
\begin{aligned}
\left.\partial_{x^{\prime}} \partial_{x_{n}} \sigma_{-2}\left(D^{-2}\right)\left(x_{0}\right)\right|_{\left|\xi^{\prime}\right|=1} & \\
= & \frac{-1}{\left(1+\xi_{n}^{2}\right)^{2}} \partial_{x_{i}} \partial_{x_{n}}\left(|\xi|^{2}\right)\left(x_{0}\right) \\
& +\frac{2}{\left(1+\xi_{n}^{2}\right)^{3}} \partial_{x_{n}}\left(|\xi|^{2}\right) \partial_{x_{i}}\left(|\xi|^{2}\right)\left(x_{0}\right)=0
\end{aligned}
$$

Therefore, Case 5 vanishes.

Case 6. Consider $r=-2, \ell=-2, k=2, \quad j=0$, and $|\alpha|=0$. From (23), we have

Case 6

$$
\begin{gathered}
=\frac{i}{6} \int_{\left|\xi^{\prime}\right|=1} \int_{-\infty}^{+\infty} \sum_{k=2} \operatorname{trace}\left[\partial_{\xi_{n}}^{2} \pi_{\xi_{n}}^{+} \sigma_{-2}\left(D^{-2}\right) \partial_{\xi_{n}}\right. \\
\left.\times \partial_{x_{n}}^{2} \sigma_{-2}\left(D^{-2}\right)\right] \\
\times\left(x_{0}\right) \mathrm{d} \xi_{n} \sigma\left(\xi^{\prime}\right) \mathrm{d} x^{\prime} .
\end{gathered}
$$

From (50)-(53), we have

$$
\begin{gathered}
\left.\partial_{\xi_{n}}^{2} \pi_{\xi_{n}}^{+} \sigma_{-2}\left(D^{-2}\right)\left(x_{0}\right)\right|_{\left|\xi^{\prime}\right|=1}=\frac{-i}{\left(\xi_{n}-i\right)^{3}} \\
\left.\partial_{\xi_{n}} \partial_{x_{n}}^{2} \sigma_{-2}\left(D^{-2}\right)\left(x_{0}\right)\right|_{\left|\xi^{\prime}\right|=1}=\frac{4 \xi_{n} h^{\prime \prime}\left(x_{0}\right)}{\left(1+\xi_{n}^{2}\right)^{3}}+\frac{-12 \xi_{n}\left(h^{\prime}(0)\right)^{2}}{\left(1+\xi_{n}^{2}\right)^{4}}
\end{gathered}
$$


Therefore,

Case 6

$$
\begin{aligned}
= & \frac{i}{6} h^{\prime \prime}(0) \\
& \times \int_{\left|\xi^{\prime}\right|=1} \int_{-\infty}^{+\infty} \frac{-32 i \xi_{n}}{\left(\xi_{n}-i\right)^{3}\left(1+\xi_{n}^{2}\right)^{3}} \mathrm{~d} \xi_{n} \sigma\left(\xi^{\prime}\right) \mathrm{d} x^{\prime} \\
& +\frac{i}{6}\left(h^{\prime}(0)\right)^{2} \\
& \times \int_{\left|\xi^{\prime}\right|=1} \int_{-\infty}^{+\infty} \frac{96 i \xi_{n}}{\left(\xi_{n}-i\right)^{3}\left(1+\xi_{n}^{2}\right)^{4}} \mathrm{~d} \xi_{n} \sigma\left(\xi^{\prime}\right) \mathrm{d} x^{\prime} \\
= & \left.\frac{i}{6} h^{\prime \prime}(0) \frac{2 \pi i}{5 !}\left[\frac{-32 i \xi_{n}}{\left(\xi_{n}+i\right)^{3}}\right]^{(5)}\right|_{\xi_{n}=i} \Omega_{5} \mathrm{~d} x^{\prime} \\
& +\left.\frac{i}{6}\left(h^{\prime}(0)\right)^{2} \frac{2 \pi i}{6 !}\left[\frac{96 i \xi_{n}}{\left(\xi_{n}+i\right)^{4}}\right]^{(6)}\right|_{\xi_{n}=i} \Omega_{5} \mathrm{~d} x^{\prime} \\
= & \left(-\frac{3}{8} h^{\prime \prime}(0)+\frac{7}{8}\left(h^{\prime}(0)\right)^{2}\right) \pi \Omega_{5} \mathrm{~d} x^{\prime} .
\end{aligned}
$$

Case 7. Consider $r=-2, \ell=-3, k=0, j=1$, and $|\alpha|=0$.

From (23) and the Leibniz rule, we obtain

Case 7

$$
\begin{gathered}
=\frac{1}{2} \int_{\left|\xi^{\prime}\right|=1} \int_{-\infty}^{+\infty} \operatorname{trace}\left[\partial_{\xi_{n}} \partial_{x_{n}} \pi_{\xi_{n}}^{+} \sigma_{-2}\right. \\
\left.\times\left(D^{-2}\right) \partial_{\xi_{n}} \sigma_{-3}\left(D^{-2}\right)\right] \\
\times\left(x_{0}\right) \mathrm{d} \xi_{n} \sigma\left(\xi^{\prime}\right) \mathrm{d} x^{\prime} \\
=-\frac{1}{2} \int_{\left|\xi^{\prime}\right|=1} \int_{-\infty}^{+\infty} \operatorname{trace}\left[\partial_{\xi_{n}}^{2} \partial_{x_{n}} \pi_{\xi_{n}}^{+} \sigma_{-2}\right. \\
\left.\times\left(D^{-2}\right) \sigma_{-3}\left(D^{-2}\right)\right] \\
\times\left(x_{0}\right) \mathrm{d} \xi_{n} \sigma\left(\xi^{\prime}\right) \mathrm{d} x^{\prime} .
\end{gathered}
$$

We note that $\int_{\left|\xi^{\prime}\right|=1} \xi_{1} \cdots \xi_{2 q+1} \sigma\left(\xi^{\prime}\right)=0$, so the first term in (79) has no contribution for computing Case 7. Combining (78), (79), and direct computations, we obtain

$$
\begin{array}{r}
\operatorname{trace}\left[\partial_{\xi_{n}}^{2} \partial_{x_{n}} \pi_{\xi_{n}}^{+} \sigma_{-2}\left(D^{-2}\right) \sigma_{-3}\left(D^{-2}\right)\right]\left(x_{0}\right) \\
=\left(h^{\prime}(0)\right)^{2} \frac{-80 i \xi_{n}+20 \xi_{n}^{2}-48 i \xi_{n}^{3}+12 \xi_{n}^{4}}{\left(\xi_{n}-i\right)^{4}\left(1+\xi_{n}^{2}\right)^{3}} .
\end{array}
$$

Therefore,

Case 7

$$
\begin{aligned}
=-\frac{1}{2}\left(h^{\prime}(0)\right)^{2} & \\
\times \int_{\left|\xi^{\prime}\right|=1} \int_{-\infty}^{+\infty}( & \left(-80 i \xi_{n}+20 \xi_{n}^{2}-48 i \xi_{n}^{3}+12 \xi_{n}^{4}\right) \\
& \left.\times\left(\left(\xi_{n}-i\right)^{4}\left(1+\xi_{n}^{2}\right)^{3}\right)^{-1}\right) \mathrm{d} \xi_{n} \sigma\left(\xi^{\prime}\right) \mathrm{d} x^{\prime}
\end{aligned}
$$

$$
\left.\pi_{\xi_{n}}^{+} \partial_{x_{n}} \sigma_{-2}\left(D^{-2}\right)\left(x_{0}\right)\right|_{\left|\xi^{\prime}\right|=1}=h^{\prime}(0) \frac{2+i \xi_{n}}{4\left(\xi_{n}-i\right)^{2}} .
$$

Then,

$$
\left.\partial_{\xi_{n}}^{2} \pi_{\xi_{n}}^{+} \partial_{x_{n}} \sigma_{-2}\left(D^{-2}\right)\left(x_{0}\right)\right|_{\left|\xi^{\prime}\right|=1}=h^{\prime}(0) \frac{4+i \xi_{n}}{2\left(\xi_{n}-i\right)^{4}} .
$$

$$
\begin{aligned}
= & -\frac{1}{2}\left(h^{\prime}(0)\right)^{2} \frac{2 \pi i}{6 !} \\
& \times\left.\left[\frac{-80 i \xi_{n}+20 \xi_{n}^{2}-48 i \xi_{n}^{3}+12 \xi_{n}^{4}}{\left(\xi_{n}+i\right)^{3}}\right]^{(6)}\right|_{\xi_{n}=i} \Omega_{5} \mathrm{~d} x^{\prime} \\
= & \frac{21}{8}\left(h^{\prime}(0)\right)^{2} \pi \Omega_{5} \mathrm{~d} x^{\prime} .
\end{aligned}
$$


Case 8. Consider $r=-2, \ell=-3, k=0, j=0$, and $|\alpha|=1$. From (23) and the Leibniz rule, we obtain

Case 8

$$
\begin{gathered}
=-\int_{\left|\xi^{\prime}\right|=1} \int_{-\infty}^{+\infty} \sum_{|\alpha|=1} \operatorname{trace}\left[\partial_{\xi^{\prime}}^{\alpha} \pi_{\xi_{n}}^{+} \sigma_{-2}\left(D^{-2}\right)\right. \\
\left.\times \partial_{x^{\prime}}^{\alpha} \partial_{\xi_{n}} \sigma_{-3}\left(D^{-2}\right)\right] \\
\times\left(x_{0}\right) \mathrm{d} \xi_{n} \sigma\left(\xi^{\prime}\right) \mathrm{d} x^{\prime} \\
=\int_{\left|\xi^{\prime}\right|=1} \int_{-\infty}^{+\infty} \sum_{|\alpha|=1} \operatorname{trace}\left[\partial_{\xi_{n}} \partial_{\xi^{\prime}}^{\alpha} \pi_{\xi_{n}}^{+} \sigma_{-2}\left(D^{-2}\right)\right. \\
\left.\times \partial_{x^{\prime}}^{\alpha} \sigma_{-3}\left(D^{-2}\right)\right] \\
\times\left(x_{0}\right) \mathrm{d} \xi_{n} \sigma\left(\xi^{\prime}\right) \mathrm{d} x^{\prime} .
\end{gathered}
$$

By Lemma 7, a simple computation shows

$$
\begin{aligned}
\left.\partial_{\xi^{\prime}}^{\alpha} \sigma_{-2}\left(D^{-2}\right)\left(x_{0}\right)\right|_{\left|\xi^{\prime}\right|=1} & =\left.\partial_{\xi_{i}} \sigma_{-2}\left(D^{-2}\right)\left(x_{0}\right)\right|_{\left|\xi^{\prime}\right|=1} \\
& =\frac{-2 \xi_{i}}{\left(1+\xi_{n}^{2}\right)^{2}} .
\end{aligned}
$$

From (53) and (83), we obtain

$$
\begin{aligned}
\left.\partial_{\xi_{n}} \partial_{\xi^{\prime}}^{\alpha} \pi_{\xi_{n}}^{+} \sigma_{-2}\left(D^{-2}\right)\left(x_{0}\right)\right|_{\left|\xi^{\prime}\right|=1} & =\left.\partial_{\xi_{i}} \sigma_{-2}\left(D^{-2}\right)\left(x_{0}\right)\right|_{\left|\xi^{\prime}\right|=1} \\
& =\frac{-3-i \xi_{n}}{2\left(\xi_{n}-i\right)^{3}} \xi_{i} .
\end{aligned}
$$

By (44), (84), and direct computations, we obtain

$$
\begin{aligned}
\operatorname{trace} & {\left[\partial_{\xi_{n}} \partial_{\xi^{\prime}}^{\alpha} \pi_{\xi_{n}}^{+} \sigma_{-2}\left(D^{-2}\right) \partial_{x^{\prime}}^{\alpha} \sigma_{-3}\left(D^{-2}\right)\right]\left(x_{0}\right) } \\
= & \frac{8 \xi_{n}-24 i}{3\left(\xi_{n}-i\right)^{3}\left(1+\xi_{n}^{2}\right)^{3}} \\
& \times \sum_{\alpha, \beta<n}\left(R_{i \alpha j \beta}^{\partial_{M}}\left(x_{0}\right)+R_{i \beta j \alpha}^{\partial_{M}}\left(x_{0}\right)\right) \xi_{i} \xi_{j} \xi_{\alpha} \xi_{\beta} \\
& +\frac{30 i-10 \xi_{n}}{3\left(\xi_{n}-i\right)^{3}\left(1+\xi_{n}^{2}\right)^{2}} \sum_{i<n} R_{i \gamma_{i k}}^{\partial_{M}}\left(x_{0}\right) \xi_{k} \xi_{\gamma} .
\end{aligned}
$$

From (63), (64), and (85), we obtain

$$
\text { Case } \begin{aligned}
8 & =\frac{1}{9} s_{\partial_{M}} \Omega_{5} \int_{-\infty}^{+\infty} \frac{15 i-5 \xi_{n}}{\left(\xi_{n}-i\right)^{3}\left(1+\xi_{n}^{2}\right)^{2}} \mathrm{~d} \xi_{n} \mathrm{~d} x^{\prime} \\
& =\left.\frac{1}{9} s_{\partial_{M}} \frac{2 \pi i}{4 !}\left[\frac{15 i-5 \xi_{n}}{\left(\xi_{n}+i\right)^{2}}\right]^{(4)}\right|_{\xi_{n}=i} \Omega_{5} \mathrm{~d} x^{\prime} \\
& =\frac{5}{16} s_{\partial_{M}} \pi \Omega_{5} \mathrm{~d} x^{\prime} .
\end{aligned}
$$

Case 9. Consider $r=-2, \ell=-3, k=1, j=0$, and $|\alpha|=0$. From (23) and the Leibniz rule, we obtain

\section{Case 9}

$$
\begin{gathered}
=-\frac{1}{2} \int_{\left|\xi^{\prime}\right|=1} \int_{-\infty}^{+\infty} \sum_{|\alpha|=1} \operatorname{trace}\left[\partial_{\xi_{n}} \pi_{\xi_{n}}^{+} \sigma_{-2}\left(D^{-2}\right)\right. \\
\left.\times \partial_{\xi_{n}} \partial_{x_{n}} \sigma_{-3}\left(D^{-2}\right)\right] \\
\times\left(x_{0}\right) \mathrm{d} \xi_{n} \sigma\left(\xi^{\prime}\right) \mathrm{d} x^{\prime} \\
=\frac{1}{2} \int_{\left|\xi^{\prime}\right|=1} \int_{-\infty}^{+\infty} \sum_{|\alpha|=1} \operatorname{trace}\left[\partial_{\xi_{n}}^{2} \pi_{\xi_{n}}^{+} \sigma_{-2}\left(D^{-2}\right) \partial_{x_{n}} \sigma_{-3}\left(D^{-2}\right)\right] \\
\times\left(x_{0}\right) \mathrm{d} \xi_{n} \sigma\left(\xi^{\prime}\right) \mathrm{d} x^{\prime} .
\end{gathered}
$$

From (73), we have

$$
\left.\partial_{\xi_{n}}^{2} \pi_{\xi_{n}}^{+} \sigma_{-2}\left(D^{-2}\right)\left(x_{0}\right)\right|_{\left|\xi^{\prime}\right|=1}=\frac{-i}{\left(\xi_{n}-i\right)^{3}} .
$$

Combining (45) and (88), we obtain

$$
\begin{gathered}
\left.\operatorname{trace}\left[\partial_{\xi_{n}}^{2} \pi_{\xi_{n}}^{+} \sigma_{-2}\left(D^{-2}\right) \partial_{x_{n}} \sigma_{-3}\left(D^{-2}\right)\right]\left(x_{0}\right)\right|_{\left|\xi^{\prime}\right|=1} \\
=\frac{\left(h^{\prime}(0)\right)^{2}\left(84 \xi_{n}+36 \xi_{n}^{3}\right)}{\left(\xi_{n}-i\right)^{3}\left(1+\xi_{n}^{2}\right)^{3}}+\frac{-24 \xi_{n} h^{\prime \prime}(0)}{\left(\xi_{n}-i\right)^{3}\left(1+\xi_{n}^{2}\right)^{2}} .
\end{gathered}
$$

Therefore,

Case 9

$$
\begin{aligned}
= & \frac{1}{2}\left(h^{\prime}(0)\right)^{2} \\
& \times \int_{\left|\xi^{\prime}\right|=1} \int_{-\infty}^{+\infty} \frac{\left(84 \xi_{n}+36 \xi_{n}^{3}\right)}{\left(\xi_{n}-i\right)^{3}\left(1+\xi_{n}^{2}\right)^{3}} \mathrm{~d} \xi_{n} \sigma\left(\xi^{\prime}\right) \mathrm{d} x^{\prime} \\
& +\frac{1}{2} h^{\prime \prime}(0) \\
& \times \int_{\left|\xi^{\prime}\right|=1} \int_{-\infty}^{+\infty} \frac{-24 \xi_{n}}{\left(\xi_{n}-i\right)^{3}\left(1+\xi_{n}^{2}\right)^{2}} \mathrm{~d} \xi_{n} \sigma\left(\xi^{\prime}\right) \mathrm{d} x^{\prime} \\
= & \frac{1}{2}\left(h^{\prime}(0)\right)^{2} \Omega_{5} \int_{\Gamma^{+}} \frac{84 \xi_{n}+36 \xi_{n}^{3}}{\left(\xi_{n}-i\right)^{3}\left(1+\xi_{n}^{2}\right)^{3}} \mathrm{~d} \xi_{n} \mathrm{~d} x^{\prime} \\
& +\frac{1}{2} h^{\prime \prime}(0) \Omega_{5} \int_{\Gamma^{+}} \frac{-24 \xi_{n}}{\left(\xi_{n}-i\right)^{3}\left(1+\xi_{n}^{2}\right)^{2}} \mathrm{~d} \xi_{n} \mathrm{~d} x^{\prime} \\
= & \left.\frac{1}{2}\left(h^{\prime}(0)\right)^{2} \frac{2 \pi i}{5 !}\left[\frac{84 \xi_{n}+36 \xi_{n}^{3}}{\left(\xi_{n}+i\right)^{3}}\right]^{(5)}\right|_{\xi_{n}=i} \Omega_{5} \mathrm{~d} x^{\prime} \\
& +\left.\frac{1}{2} h^{\prime \prime}(0) \frac{2 \pi i}{4 !}\left[\frac{-24 \xi_{n}}{\left(\xi_{n}+i\right)^{2}}\right]^{(4)}\right|_{\xi_{n}=i} \Omega_{5} \mathrm{~d} x^{\prime} \\
= & \left(\frac{9}{8} h^{\prime \prime}(0)-\frac{27}{8}\left(h^{\prime}(0)\right)^{2}\right) \pi \Omega_{5} \mathrm{~d} x^{\prime} .
\end{aligned}
$$


Case 10. Consider $r=-3, \ell=-2, k=0, j=1$, and $|\alpha|=0$.

From (23), we have

Case 10

$$
\begin{gathered}
=-\frac{1}{2} \int_{\left|\xi^{\prime}\right|=1} \int_{-\infty}^{+\infty} \operatorname{trace}\left[\partial_{x_{n}} \pi_{\xi_{n}}^{+} \sigma_{-3}\left(D^{-2}\right) \partial_{\xi_{n}}^{2} \sigma_{-2}\left(D^{-2}\right)\right] \\
\times\left(x_{0}\right) \mathrm{d} \xi_{n} \sigma\left(\xi^{\prime}\right) \mathrm{d} x^{\prime} .
\end{gathered}
$$

By the Leibniz rule, trace property, and “++" and “- -" vanishing after the integration over $\xi_{n}$ in [9], then

$$
\begin{gathered}
\int_{-\infty}^{+\infty} \operatorname{trace}\left[\partial_{x_{n}} \pi_{\xi_{n}}^{+} \sigma_{-3}\left(D^{-2}\right) \partial_{\xi_{n}}^{2} \sigma_{-2}\left(D^{-2}\right)\right] \mathrm{d} \xi_{n} \\
=\int_{-\infty}^{+\infty} \operatorname{trace}\left[\partial_{x_{n}} \sigma_{-3}\left(D^{-2}\right) \partial_{\xi_{n}}^{2} \sigma_{-2}\left(D^{-2}\right)\right] \mathrm{d} \xi_{n} \\
\quad-\int_{-\infty}^{+\infty} \operatorname{trace}\left[\partial_{x_{n}} \sigma_{-3}\left(D^{-2}\right) \partial_{\xi_{n}}^{2} \pi_{\xi_{n}}^{+} \sigma_{-2}\left(D^{-2}\right)\right] \mathrm{d} \xi_{n} .
\end{gathered}
$$

Combining these assertions, we obtain

Case 10

$=$ Case 9

$$
\begin{aligned}
-\frac{1}{2} \int_{\left|\xi^{\prime}\right|=1} \int_{-\infty}^{+\infty} \operatorname{trace} & {\left[\partial_{x_{n}} \sigma_{-3}\left(D^{-2}\right)\right.} \\
& \left.\times \partial_{\xi_{n}}^{2} \sigma_{-2}\left(D^{-2}\right)\right] \mathrm{d} \xi_{n} \sigma\left(\xi^{\prime}\right) \mathrm{d} x^{\prime}
\end{aligned}
$$

By Lemma 7, a simple computation shows

$$
\left.\partial_{\xi_{n}}^{2} \sigma_{-2}\left(D^{-2}\right)\left(x_{0}\right)\right|_{\left|\xi^{\prime}\right|=1}=\frac{6 \xi_{n}^{2}-2}{\left(1+\xi_{n}^{2}\right)^{3}} .
$$

Combining (45) and (94), we obtain

$$
\begin{aligned}
\operatorname{trace} & {\left[\partial_{x_{n}} \sigma_{-3}\left(D^{-2}\right) \partial_{\xi_{n}}^{2} \sigma_{-2}\left(D^{-2}\right)\right]\left(x_{0}\right) } \\
= & \left(h^{\prime}(0)\right)^{2} \frac{\left(84 i \xi_{n}+36 i \xi_{n}^{3}\right)\left(-2+6 \xi_{n}^{2}\right)}{\left(1+\xi_{n}^{2}\right)^{6}} \\
& +h^{\prime \prime}(0) \frac{24 i \xi_{n}\left(2-6 \xi_{n}^{2}\right)}{\left(1+\xi_{n}^{2}\right)^{5}} .
\end{aligned}
$$

We note that

$$
\begin{gathered}
\int_{-\infty}^{+\infty} \frac{\left(84 i \xi_{n}+36 i \xi_{n}^{3}\right)\left(-2+6 \xi_{n}^{2}\right)}{\left(1+\xi_{n}^{2}\right)^{6}} \mathrm{~d} \xi_{n} \\
=\left.\frac{2 \pi i}{5 !}\left[\frac{\left(84 i \xi_{n}+36 i \xi_{n}^{3}\right)\left(-2+6 \xi_{n}^{2}\right)}{\left(\xi_{n}+i\right)^{6}}\right]^{(5)}\right|_{\xi_{n}=i}=0, \\
\int_{-\infty}^{+\infty} \frac{24 i \xi_{n}\left(2-6 \xi_{n}^{2}\right)}{\left(1+\xi_{n}^{2}\right)^{5}} \mathrm{~d} \xi_{n}=0 .
\end{gathered}
$$

Therefore,

$$
\text { Case } 10=\left(\frac{9}{8} h^{\prime \prime}(0)-\frac{27}{8}\left(h^{\prime}(0)\right)^{2}\right) \pi \Omega_{5} \mathrm{~d} x^{\prime} .
$$

Case 11. Consider $r=-3, \ell=-2, k=0, j=0$, and $|\alpha|=1$. From (23), we have

\section{Case 11}

$$
\begin{gathered}
=-\int_{\left|\xi^{\prime}\right|=1} \int_{-\infty}^{+\infty} \sum_{|\alpha|=1} \operatorname{trace}\left[\partial_{\xi^{\prime}}^{\alpha} \pi_{\xi_{n}}^{+} \sigma_{-3}\left(D^{-2}\right)\right. \\
\left.\times \partial_{x^{\prime}}^{\alpha} \partial_{\xi_{n}} \sigma_{-2}\left(D^{-2}\right)\right] \\
\times\left(x_{0}\right) \mathrm{d} \xi_{n} \sigma\left(\xi^{\prime}\right) \mathrm{d} x^{\prime} .
\end{gathered}
$$

By Lemma 8 , for $i<n$, we have

$$
\partial_{x_{i}} \sigma_{-2}\left(D^{-2}\right)\left(x_{0}\right)=\partial_{x_{i}}\left(|\xi|^{-2}\right)\left(x_{0}\right)=0 .
$$

\section{So Case 11 vanishes.}

Case 12. Consider $r=-3, \ell=-2, k=1, j=0$, and $|\alpha|=0$.

From (23) and the Leibniz rule, we have

Case 12

$$
\begin{aligned}
&=-\frac{1}{2} \int_{\left|\xi^{\prime}\right|=1} \int_{-\infty}^{+\infty} \operatorname{trace}\left[\partial_{x_{n}} \pi_{\xi_{n}}^{+} \sigma_{-3}\left(D^{-2}\right) \partial_{\xi_{n}} \partial_{x_{n}} \sigma_{-2}\left(D^{-2}\right)\right] \\
& \times\left(x_{0}\right) \mathrm{d} \xi_{n} \sigma\left(\xi^{\prime}\right) \mathrm{d} x^{\prime} \\
&=\frac{1}{2} \int_{\left|\xi^{\prime}\right|=1} \int_{-\infty}^{+\infty} \operatorname{trace}\left[\pi_{\xi_{n}}^{+} \sigma_{-3}\left(D^{-2}\right) \partial_{\xi_{n}}^{2} \partial_{x_{n}} \sigma_{-2}\left(D^{-2}\right)\right] \\
& \times\left(x_{0}\right) \mathrm{d} \xi_{n} \sigma\left(\xi^{\prime}\right) \mathrm{d} x^{\prime}
\end{aligned}
$$

By the Leibniz rule, trace property, and "++" and “- -" vanishing after the integration over $\xi_{n}$ in [9], then

$$
\begin{aligned}
& \int_{-\infty}^{+\infty} \operatorname{trace}\left[\pi_{\xi_{n}}^{+} \sigma_{-3}\left(D^{-2}\right) \partial_{\xi_{n}}^{2} \partial_{x_{n}} \sigma_{-2}\left(D^{-2}\right)\right] \mathrm{d} \xi_{n} \\
& =\int_{-\infty}^{+\infty} \operatorname{trace}\left[\sigma_{-3}\left(D^{-2}\right) \partial_{\xi_{n}}^{2} \partial_{x_{n}} \sigma_{-2}\left(D^{-2}\right)\right] \mathrm{d} \xi_{n} \\
& \quad-\int_{-\infty}^{+\infty} \operatorname{trace}\left[\sigma_{-3}\left(D^{-2}\right) \partial_{\xi_{n}}^{2} \partial_{x_{n}} \pi_{\xi_{n}}^{+} \sigma_{-2}\left(D^{-2}\right)\right] \mathrm{d} \xi_{n} .
\end{aligned}
$$

Combining these assertions, we see

Case $12=$ Case 7

$$
\begin{gathered}
+\frac{1}{2} \int_{\left|\xi^{\prime}\right|=1} \int_{-\infty}^{+\infty} \operatorname{trace}\left[\sigma_{-3}\left(D^{-2}\right) \partial_{\xi_{n}}^{2} \partial_{x_{n}} \sigma_{-2}\left(D^{-2}\right)\right] \\
\times\left(x_{0}\right) \mathrm{d} \xi_{n} \sigma\left(\xi^{\prime}\right) \mathrm{d} x^{\prime}
\end{gathered}
$$


From (68) and direct computations, we obtain

$$
\left.\partial_{\xi_{n}}^{2} \partial_{x_{n}} \sigma_{-2}\left(D^{-2}\right)\left(x_{0}\right)\right|_{\left|\xi^{\prime}\right|=1}=\frac{4-20 \xi_{n}^{2}}{\left(1+\xi_{n}^{2}\right)^{4}} h^{\prime}(0) .
$$

Combining (79) and (103), we obtain

$$
\begin{gathered}
\operatorname{trace}\left[\sigma_{-3}\left(D^{-2}\right) \partial_{\xi_{n}}^{2} \partial_{x_{n}} \sigma_{-2}\left(D^{-2}\right)\right]\left(x_{0}\right) \\
=\left(h^{\prime}(0)\right)^{2-20 i \xi_{n}+88 i \xi_{n}^{3}+60 i \xi_{n}^{5}} \\
\left(1+\xi_{n}^{2}\right)^{7}
\end{gathered}
$$

We note that

$$
\begin{aligned}
& \int_{-\infty}^{+\infty} \frac{-20 i \xi_{n}+88 i \xi_{n}^{3}+60 i \xi_{n}^{5}}{\left(1+\xi_{n}^{2}\right)^{7}} \mathrm{~d} \xi_{n} \\
& \quad=\left.\frac{2 \pi i}{6 !}\left[\frac{-20 i \xi_{n}+88 i \xi_{n}^{3}+60 i \xi_{n}^{5}}{\left(\xi_{n}+i\right)^{7}}\right]^{(6)}\right|_{\xi_{n}=i}=0 .
\end{aligned}
$$

Therefore,

$$
\text { Case } 12=\frac{21}{8}\left(h^{\prime}(0)\right)^{2} \pi \Omega_{5} \mathrm{~d} x^{\prime}
$$

Case 13. Consider $r=-3, \ell=-3, k=0, j=0$, and $|\alpha|=0$.

From (23) and the Leibniz rule, we have

Case 13

$$
\begin{gathered}
=-i \int_{\left|\xi^{\prime}\right|=1} \int_{-\infty}^{+\infty} \operatorname{trace}\left[\pi_{\xi_{n}}^{+} \sigma_{-3}\left(D^{-2}\right) \partial_{\xi_{n}} \sigma_{-3}\left(D^{-2}\right)\right] \\
\times\left(x_{0}\right) \mathrm{d} \xi_{n} \sigma\left(\xi^{\prime}\right) \mathrm{d} x^{\prime} \\
=i \int_{\left|\xi^{\prime}\right|=1} \int_{-\infty}^{+\infty} \operatorname{trace}\left[\partial_{\xi_{n}} \pi_{\xi_{n}}^{+} \sigma_{-3}\left(D^{-2}\right) \sigma_{-3}\left(D^{-2}\right)\right] \\
\times\left(x_{0}\right) \mathrm{d} \xi_{n} \sigma\left(\xi^{\prime}\right) \mathrm{d} x^{\prime} .
\end{gathered}
$$

By (79), we obtain

$$
\begin{aligned}
&\left.\sigma_{-3}\left(D^{-2}\right)\left(x_{0}\right)\right|_{\left|\xi^{\prime}\right|=1} \\
&=-\left.\sqrt{-1}|\xi|^{-4} \xi_{k}\left(\Gamma^{k}-2 \delta^{k}\right)\left(x_{0}\right)\right|_{\left|\xi^{\prime}\right|=1} \\
&-\left.\sqrt{-1}|\xi|^{-6} 2 \xi^{j} \xi_{\alpha} \xi_{\beta} \partial_{j} g^{\alpha \beta}\left(x_{0}\right)\right|_{\left|\xi^{\prime}\right|=1} \\
&= \frac{-i}{\left(1+\xi_{n}^{2}\right)^{2}}\left(-\frac{1}{2} h^{\prime}(0) \sum_{k<n} \xi_{k} c\left(\widetilde{e_{k}}\right) c\left(\widetilde{e_{n}}\right)+3 h^{\prime}(0) \xi_{n}\right) \\
&-\frac{2 i h^{\prime}(0) \xi_{n}}{\left(1+\xi_{n}^{2}\right)^{3}} \\
&= \frac{i}{2\left(1+\xi_{n}^{2}\right)^{2}} h^{\prime}(0) \sum_{k<n} \xi_{k} c\left(\widetilde{e_{k}}\right) c\left(\widetilde{e_{n}}\right)+h^{\prime}(0) \frac{-5 i \xi_{n}-3 i \xi_{n}^{3}}{\left(1+\xi_{n}^{2}\right)^{3}} .
\end{aligned}
$$

By (18) and the Cauchy integral formula, then

$$
\begin{aligned}
& \pi_{\xi_{n}}^{+}\left[\frac{-5 i \xi_{n}-3 i \xi_{n}^{3}}{\left(1+\xi_{n}^{2}\right)^{3}}\right] \\
& =\pi_{\xi_{n}}^{+}\left[\frac{-5 i \xi_{n}-3 i \xi_{n}^{3}}{\left(\xi_{n}+i\right)^{3}\left(\xi_{n}-i\right)^{3}}\right] \\
& =\frac{1}{2 \pi i} \lim _{u \rightarrow 0^{-}} \int_{\Gamma^{+}} \frac{\left(-5 i \eta_{n}-3 i \eta_{n}^{3}\right) /\left(\left(\eta_{n}+i\right)^{3}\left(\xi_{n}+i u-\eta_{n}\right)\right)}{\left(\eta_{n}-i\right)^{3}} \mathrm{~d} \eta_{n} \\
& =\left.\frac{1}{2}\left[\frac{-5 i \eta_{n}-3 i \eta_{n}^{3}}{\left(\eta_{n}+i\right)^{3}\left(\xi_{n}-\eta_{n}\right)}\right]^{(1)}\right|_{\eta_{n}=i}=\frac{9 i-7 \xi_{n}}{8\left(\xi_{n}-i\right)^{3}} .
\end{aligned}
$$

Then, we obtain

$$
\begin{aligned}
\left.\partial_{\xi_{n}} \pi_{\xi_{n}}^{+} \sigma_{-3}\left(D^{-2}\right)\left(x_{0}\right)\right|_{\left|\xi^{\prime}\right|=1} \\
=\frac{3 i-\xi_{n}}{4\left(\xi_{n}-i\right)^{3}} h^{\prime}(0) \sum_{k<n} \xi_{k} c\left(\widetilde{e_{k}}\right) c\left(\widetilde{e_{n}}\right) \\
+h^{\prime}(0) \frac{7 \xi_{n}-10 i}{4\left(\xi_{n}-i\right)^{4}} .
\end{aligned}
$$

By the relation of the Clifford action and $\operatorname{tr} A B=\operatorname{tr} B A$, then we have the equalities

$$
\begin{aligned}
& \operatorname{tr}\left[c\left(\xi^{\prime}\right) c\left(d x_{n}\right)\right]=0 ; \quad \operatorname{tr}\left[c\left(d x_{n}\right)^{2}\right]=-8 \\
& \left.\operatorname{tr}\left[c\left(\xi^{\prime}\right)^{2}\right]\left(x_{0}\right)\right|_{\left|\xi^{\prime}\right|=1}=-8 ; \\
& \operatorname{tr}\left[c\left(\widetilde{E_{j}}\right) c\left(\widetilde{E_{n}}\right) c\left(\widetilde{E_{k}}\right) c\left(\widetilde{E_{n}}\right)\right]=-\operatorname{tr}[\mathrm{id}] \delta_{j}^{k}=-8 \delta_{j}^{k} .
\end{aligned}
$$

Then,

$$
\begin{aligned}
\operatorname{trace} & {\left[\partial_{\xi_{n}} \pi_{\xi_{n}}^{+} \sigma_{-3}\left(D^{-2}\right) \sigma_{-3}\left(D^{-2}\right)\right]\left(x_{0}\right) } \\
= & \frac{\left(-3-i \xi_{n}\right) h^{\prime}(0)}{8\left(\xi_{n}-i\right)^{3}\left(1+\xi_{n}^{2}\right)^{2}} \\
& \times \sum_{k<n} \xi_{j} \xi_{k} \operatorname{trace}\left[c\left(\widetilde{E_{j}}\right) c\left(\widetilde{E_{n}}\right) c\left(\widetilde{E_{k}}\right) c\left(\widetilde{E_{n}}\right)\right] \\
& +\left(h^{\prime}(0)\right)^{2} \frac{2\left(10 i-7 \xi_{n}\right)\left(5 i \xi_{n}+3 i \xi_{n}^{3}\right)}{\left(\xi_{n}-i\right)^{4}\left(1+\xi_{n}^{2}\right)^{3}} \\
= & \left(h^{\prime}(0)\right)^{2-3 i-96 \xi_{n}-72 i \xi_{n}^{2}-56 \xi_{n}^{3}-41 i \xi_{n}^{4}} \\
& \left(\xi_{n}-i\right)^{4}\left(1+\xi_{n}^{2}\right)^{3}
\end{aligned}
$$


Therefore,

Case 13

$$
\begin{aligned}
= & i\left(h^{\prime}(0)\right)^{2} \\
& \times \int_{\left|\xi^{\prime}\right|=1} \int_{-\infty}^{+\infty}\left(\left(-3 i-96 \xi_{n}-72 i \xi_{n}^{2}-56 \xi_{n}^{3}-41 i \xi_{n}^{4}\right)\right. \\
& \left.\times\left(\left(\xi_{n}-i\right)^{4}\left(1+\xi_{n}^{2}\right)^{3}\right)^{-1}\right) \mathrm{d} \xi_{n} \sigma\left(\xi^{\prime}\right) \mathrm{d} x^{\prime} \\
= & i\left(h^{\prime}(0)\right)^{2} \Omega_{5} \\
& \times \int_{\Gamma^{+}} \frac{-3 i-96 \xi_{n}-72 i \xi_{n}^{2}-56 \xi_{n}^{3}-41 i \xi_{n}^{4}}{\left(\xi_{n}-i\right)^{4}\left(1+\xi_{n}^{2}\right)^{3}} \mathrm{~d} \xi_{n} \mathrm{~d} x^{\prime} \\
= & i\left(h^{\prime}(0)\right)^{2} \frac{2 \pi i}{6 !} \\
& \times\left[\frac{-3 i-96 \xi_{n}-72 i \xi_{n}^{2}-56 \xi_{n}^{3}-41 i \xi_{n}^{4}}{\left(\xi_{n}+i\right)^{3}}\right]_{\xi_{n}=i}^{(6)} \Omega_{5} \mathrm{~d} x^{\prime} \\
= & -\frac{57}{8}\left(h^{\prime}(0)\right)^{2} \pi \Omega_{5} \mathrm{~d} x^{\prime} .
\end{aligned}
$$

Case 14. Consider $r=-2, \ell=-4, k=0, j=0$, and $|\alpha|=0$. From (23) and the Leibniz rule, we have

Case 14

$$
\begin{gathered}
=-i \int_{\left|\xi^{\prime}\right|=1} \int_{-\infty}^{+\infty} \operatorname{trace}\left[\pi_{\xi_{n}}^{+} \sigma_{-2}\left(D^{-2}\right) \partial_{\xi_{n}} \sigma_{-4}\left(D^{-2}\right)\right] \\
\times\left(x_{0}\right) \mathrm{d} \xi_{n} \sigma\left(\xi^{\prime}\right) \mathrm{d} x^{\prime} \\
=i \int_{\left|\xi^{\prime}\right|=1} \int_{-\infty}^{+\infty} \operatorname{trace}\left[\partial_{\xi_{n}} \pi_{\xi_{n}}^{+} \sigma_{-2}\left(D^{-2}\right) \sigma_{-4}\left(D^{-2}\right)\right] \\
\times\left(x_{0}\right) \mathrm{d} \xi_{n} \sigma\left(\xi^{\prime}\right) \mathrm{d} x^{\prime} .
\end{gathered}
$$

From (73), we have

$$
\left.\partial_{\xi_{n}} \pi_{\xi_{n}}^{+} \sigma_{-2}\left(D^{-2}\right)\left(x_{0}\right)\right|_{\left|\xi^{\prime}\right|=1}=\frac{i}{2\left(\xi_{n}-i\right)^{2}} .
$$

From Lemmas 7 and 10, we obtain

$$
\begin{aligned}
& \left.\sigma_{-4}\left(D^{-2}\right)\left(x_{0}\right)\right|_{\left|\xi^{\prime}\right|=1} \\
& =\frac{-\left(h^{\prime}(0)\right)^{2}}{4\left(1+\xi_{n}^{2}\right)^{3}} c\left(\widetilde{E_{\mu}}\right) c\left(\widetilde{E_{n}}\right) c\left(\widetilde{E_{\nu}}\right) c\left(\widetilde{E_{n}}\right)-\frac{9\left(h^{\prime}(0)\right)^{2}}{\left(1+\xi_{n}^{2}\right)^{3}} \xi_{n}^{3} \xi_{\mu} \xi_{\nu} \\
& \quad+\frac{\left(h^{\prime}(0)\right)^{2}}{4\left(1+\xi_{n}^{2}\right)^{2}} \xi_{\mu} \xi_{\nu} c\left(\widetilde{E_{\mu}}\right) c\left(\widetilde{E_{n}}\right) c\left(\widetilde{E_{\nu}}\right) c\left(\widetilde{E_{n}}\right) \\
& \quad-\frac{1}{4\left(1+\xi_{n}^{2}\right)^{2}} s\left(x_{0}\right)
\end{aligned}
$$

$$
\begin{aligned}
& -\frac{5}{3\left(1+\xi_{n}^{2}\right)^{3}} \xi_{\mu} \xi_{\nu} \sum_{i<n} R_{i \mu i \nu}^{\partial_{M}}\left(x_{0}\right)-\frac{6}{\left(1+\xi_{n}^{2}\right)^{3}} h^{\prime \prime}(0) \xi_{n}^{2} \\
& -\frac{4}{3\left(1+\xi_{n}^{2}\right)^{4}} \xi_{\mu} \xi_{\nu} \xi_{\gamma} \xi_{\delta} \sum_{\gamma, \delta<n}\left(R_{\mu \nu \nu \delta}^{\partial_{M}}\left(x_{0}\right)+R_{\nu \gamma \mu \delta}^{\partial_{M}}\left(x_{0}\right)\right) \\
& +\frac{4 h^{\prime \prime}(0)}{\left(1+\xi_{n}^{2}\right)^{4}} \xi_{n}^{2} \\
& -\frac{1}{3\left(1+\xi_{n}^{2}\right)^{3}} \xi_{\alpha} \xi_{\beta} \sum_{\alpha, \beta<n}\left(R_{\mu \alpha \nu \beta}^{\partial_{M}}\left(x_{0}\right)+R_{\nu \beta \mu \alpha}^{\partial_{M}}\left(x_{0}\right)\right) \\
& +\frac{h^{\prime \prime}(0)}{\left(1+\xi_{n}^{2}\right)^{3}}+\frac{2+3 \xi_{n}+10 \xi_{n}^{2}+12 \xi_{n}^{3}-4 \xi_{n}^{4}+9 \xi_{n}^{5}}{\left(1+\xi_{n}^{2}\right)^{5}}\left(h^{\prime}(0)\right)^{2} .
\end{aligned}
$$

From (115), (116), and direct computations, we obtain

$$
\begin{aligned}
\operatorname{trace} & {\left.\left[\partial_{\xi_{n}} \pi_{\xi_{n}}^{+} \sigma_{-2}\left(D^{-2}\right) \sigma_{-4}\left(D^{-2}\right)\right]\left(x_{0}\right)\right|_{\left|\xi^{\prime}\right|=1} } \\
= & \frac{i}{2\left(\xi_{n}-i\right)^{2}} \times \frac{-\left(h^{\prime}(0)\right)^{2}}{4\left(1+\xi_{n}^{2}\right)^{3}} \operatorname{tr}\left[c\left(\widetilde{E_{\mu}}\right) c\left(\widetilde{E_{n}}\right) c\left(\widetilde{E_{\nu}}\right) c\left(\widetilde{E_{n}}\right)\right] \\
& -\frac{i}{2\left(\xi_{n}-i\right)^{2}} \times \frac{9\left(h^{\prime}(0)\right)^{2}}{\left(1+\xi_{n}^{2}\right)^{3}} \xi_{n}^{3} \xi_{\mu} \xi_{\nu} \operatorname{tr}[\mathrm{id}] \\
& +\frac{i}{2\left(\xi_{n}-i\right)^{2}} \\
& \times \frac{\left(h^{\prime}(0)\right)^{2}}{4\left(1+\xi_{n}^{2}\right)^{2}} \xi_{\mu} \xi_{\nu} \operatorname{tr}\left[c\left(\widetilde{E_{\mu}}\right) c\left(\widetilde{E_{n}}\right) c\left(\widetilde{E_{\nu}}\right) c\left(\widetilde{E_{n}}\right)\right] \\
& -\frac{i}{2\left(\xi_{n}-i\right)^{2}} \times \frac{1}{4\left(1+\xi_{n}^{2}\right)^{2}} s\left(x_{0}\right) \operatorname{tr}[\mathrm{id}] \\
& -\frac{i}{2\left(\xi_{n}-i\right)^{2}} \times \frac{5}{3\left(1+\xi_{n}^{2}\right)^{3}} \xi_{\mu} \xi_{\nu} \sum_{i<n} R_{i \mu i \nu}^{\partial_{M}}\left(x_{0}\right) \operatorname{tr}[\mathrm{id}] \\
& -\frac{i}{2\left(\xi_{n}-i\right)^{2}} \times \frac{6}{\left(1+\xi_{n}^{2}\right)^{3}} h^{\prime \prime}(0) \xi_{n}^{2} \operatorname{tr}[\mathrm{id}] \\
& -\frac{i}{2\left(\xi_{n}-i\right)^{2}} \times \frac{4}{3\left(1+\xi_{n}^{2}\right)^{4}} \xi_{\mu} \xi_{\nu} \xi_{\gamma} \xi_{\delta} \\
& \times \sum_{\gamma, \delta<n}\left(R_{\mu \nu \nu \delta}^{\partial_{M}}\left(x_{0}\right)+R_{\nu \gamma \mu \delta}^{\partial_{M}}\left(x_{0}\right)\right) \operatorname{tr}[\mathrm{id}] \\
& -\frac{i}{2\left(\xi_{n}-i\right)^{2}} \times \frac{1}{3\left(1+\xi_{n}^{2}\right)^{3}} \xi_{\alpha} \xi_{\beta} \\
& \times \sum_{\alpha, \beta<n}\left(R_{\mu \alpha \nu \beta}^{\partial_{M}}\left(x_{0}\right)+R_{\nu \beta \mu \alpha}^{\partial_{M}}\left(x_{0}\right)\right) \operatorname{tr}[\mathrm{id}] \\
& \\
& \\
&
\end{aligned}
$$




$$
\begin{aligned}
& +\frac{i}{2\left(\xi_{n}-i\right)^{2}} \times \frac{4 h^{\prime \prime}(0)}{\left(1+\xi_{n}^{2}\right)^{4}} \xi_{n}^{2} \operatorname{tr}[\mathrm{id}] \\
& +\frac{i}{2\left(\xi_{n}-i\right)^{2}} \times \frac{h^{\prime \prime}(0)}{\left(1+\xi_{n}^{2}\right)^{3}} \operatorname{tr}[\mathrm{id}] \\
& +\frac{i}{2\left(\xi_{n}-i\right)^{2}} \times \frac{2+3 \xi_{n}+10 \xi_{n}^{2}+12 \xi_{n}^{3}-4 \xi_{n}^{4}+9 \xi_{n}^{5}}{\left(1+\xi_{n}^{2}\right)^{5}} \\
& \times\left(h^{\prime}(0)\right)^{2} \operatorname{tr}[\mathrm{id}] .
\end{aligned}
$$

Combining (64), (113), and (117), we obtain

Case 14

$$
\begin{aligned}
& =s\left(x_{0}\right) \Omega_{5} \int_{-\infty}^{+\infty} \frac{1}{\left(\xi_{n}-i\right)^{4}\left(\xi_{n}+i\right)^{2}} \mathrm{~d} \xi_{n} \mathrm{~d} x^{\prime} \\
& +s_{\partial_{M}}\left(x_{0}\right) \Omega_{5} \int_{-\infty}^{+\infty} \frac{14}{9\left(\xi_{n}-i\right)^{5}\left(\xi_{n}+i\right)^{3}} \mathrm{~d} \xi_{n} \mathrm{~d} x^{\prime} \\
& +\left(h^{\prime}(0)\right)^{2} \Omega_{5} \\
& \times \int_{-\infty}^{+\infty}\left(\left(-53-72 \xi_{n}-33 \xi_{n}^{2}-288 \xi_{n}^{3}+525 \xi_{n}^{4}-216 \xi_{n}^{5}\right.\right. \\
& \left.\left.+217 \xi_{n}^{6}\right) \times\left(6\left(\xi_{n}-i\right)^{7}\left(\xi_{n}+i\right)^{5}\right)^{-1}\right) \mathrm{d} \xi_{n} \mathrm{~d} x^{\prime} \\
& +h^{\prime \prime}(0) \Omega_{5} \int_{-\infty}^{+\infty} \frac{-4\left(1-\xi_{n}^{2}-6 \xi_{n}^{4}\right)}{\left(\xi_{n}-i\right)^{6}\left(\xi_{n}+i\right)^{4}} \mathrm{~d} \xi_{n} \mathrm{~d} x^{\prime} \\
& =\left.s\left(x_{0}\right) \frac{2 \pi i}{3 !}\left[\frac{1}{\left(\xi_{n}+i\right)^{2}}\right]^{(3)}\right|_{\xi_{n}=i} \Omega_{5} \mathrm{~d} x^{\prime} \\
& +\left.s_{\partial_{M}}\left(x_{0}\right) \frac{2 \pi i}{4 !}\left[\frac{14}{9\left(\xi_{n}+i\right)^{3}}\right]^{(4)}\right|_{\xi_{n}=i} \Omega_{5} \mathrm{~d} x^{\prime} \\
& +\left(h^{\prime}(0)\right)^{2} \frac{2 \pi i}{6 !} \\
& \times\left[\left(-53-72 \xi_{n}-33 \xi_{n}^{2}-288 \xi_{n}^{3}+525 \xi_{n}^{4}\right.\right. \\
& \left.\left.-216 \xi_{n}^{5}+217 \xi_{n}^{6}\right) \times\left(6\left(\xi_{n}+i\right)^{5}\right)^{-1}\right]\left.^{(6)}\right|_{\xi_{n}=i} \Omega_{5} \mathrm{~d} x^{\prime} \\
& +\left.h^{\prime \prime}(0) \frac{2 \pi i}{5 !}\left[\frac{-4\left(1-\xi_{n}^{2}-6 \xi_{n}^{4}\right)}{\left(\xi_{n}+i\right)^{4}}\right]^{(5)}\right|_{\xi_{n}=i} \Omega_{5} \mathrm{~d} x^{\prime}
\end{aligned}
$$

$$
\begin{aligned}
=\left(\frac{-1}{4}\right. & s\left(x_{0}\right)-\frac{35}{96} s_{\partial_{M}}\left(x_{0}\right) \\
& \left.+\left(\frac{343}{192}-\frac{3 i}{2}\right)\left(h^{\prime}(0)\right)^{2}+\frac{13}{16} h^{\prime \prime}(0)\right) \pi \Omega_{5} \mathrm{~d} x^{\prime} .
\end{aligned}
$$

Case 15. Consider $r=-4, \ell=-2, k=0, j=0$, and $|\alpha|=0$. From (23), we have

Case 15

$$
\begin{gathered}
=-i \int_{\left|\xi^{\prime}\right|=1} \int_{-\infty}^{+\infty} \operatorname{trace}\left[\pi_{\xi_{n}}^{+} \sigma_{-4}\left(D^{-2}\right) \partial_{\xi_{n}} \sigma_{-2}\left(D^{-2}\right)\right] \\
\times\left(x_{0}\right) \mathrm{d} \xi_{n} \sigma\left(\xi^{\prime}\right) \mathrm{d} x^{\prime} .
\end{gathered}
$$

By the Leibniz rule, trace property, and "++" and "- -" vanishing after the integration over $\xi_{n}$ in [9], then

$$
\begin{aligned}
& \int_{-\infty}^{+\infty} \operatorname{trace}\left[\pi_{\xi_{n}}^{+} \sigma_{-4}\left(D^{-2}\right) \partial_{\xi_{n}} \sigma_{-2}\left(D^{-2}\right)\right] \mathrm{d} \xi_{n} \\
& =\int_{-\infty}^{+\infty} \operatorname{trace}\left[\sigma_{-4}\left(D^{-2}\right) \partial_{\xi_{n}} \sigma_{-2}\left(D^{-2}\right)\right] \mathrm{d} \xi_{n} \\
& \quad-\int_{-\infty}^{+\infty} \operatorname{trace}\left[\sigma_{-4}\left(D^{-2}\right) \partial_{\xi_{n}} \pi_{\xi_{n}}^{+} \sigma_{-2}\left(D^{-2}\right)\right] \mathrm{d} \xi_{n} .
\end{aligned}
$$

Combining these assertions, we see

Case $15=$ Case 14

$$
\begin{gathered}
-i \int_{\left|\xi^{\prime}\right|=1} \int_{-\infty}^{+\infty} \operatorname{trace}\left[\sigma_{-4}\left(D^{-2}\right) \partial_{\xi_{n}} \sigma_{-2}\left(D^{-2}\right)\right] \\
\times\left(x_{0}\right) \mathrm{d} \xi_{n} \sigma\left(\xi^{\prime}\right) \mathrm{d} x^{\prime} .
\end{gathered}
$$

By Lemma 7, a simple computation shows

$$
\left.\partial_{\xi_{n}} \sigma_{-2}\left(D^{-2}\right)\left(x_{0}\right)\right|_{\left|\xi^{\prime}\right|=1}=\frac{-2 \xi_{n}}{\left(1+\xi_{n}^{2}\right)^{2}} .
$$

From (116), (122), and direct computations, we obtain

$$
\begin{aligned}
\operatorname{trace} & {\left.\left[\sigma_{-4}\left(D^{-2}\right) \partial_{\xi_{n}} \sigma_{-2}\left(D^{-2}\right)\right]\left(x_{0}\right)\right|_{\left|\xi^{\prime}\right|=1} } \\
= & \frac{-2 \xi_{n}}{\left(1+\xi_{n}^{2}\right)^{2}} \times \frac{-\left(h^{\prime}(0)\right)^{2}}{4\left(1+\xi_{n}^{2}\right)^{3}} \operatorname{tr}\left[c\left(\widetilde{E_{\mu}}\right) c\left(\widetilde{E_{n}}\right) c\left(\widetilde{E_{v}}\right) c\left(\widetilde{E_{n}}\right)\right] \\
& -\frac{-2 \xi_{n}}{\left(1+\xi_{n}^{2}\right)^{2}} \times \frac{9\left(h^{\prime}(0)\right)^{2}}{\left(1+\xi_{n}^{2}\right)^{3}} \xi_{n}^{3} \xi_{\mu} \xi_{\nu} \operatorname{tr}[\mathrm{id}] \\
& +\frac{-2 \xi_{n}}{\left(1+\xi_{n}^{2}\right)^{2}} \times \frac{\left(h^{\prime}(0)\right)^{2}}{4\left(1+\xi_{n}^{2}\right)^{2}} \xi_{\mu} \xi_{v}
\end{aligned}
$$




$$
\begin{aligned}
& \times \operatorname{tr}\left[c\left(\widetilde{E_{\mu}}\right) c\left(\widetilde{E_{n}}\right) c\left(\widetilde{E_{\nu}}\right) c\left(\widetilde{E_{n}}\right)\right] \\
& -\frac{-2 \xi_{n}}{\left(1+\xi_{n}^{2}\right)^{2}} \times \frac{1}{4\left(1+\xi_{n}^{2}\right)^{2}} s\left(x_{0}\right) \operatorname{tr}[\mathrm{id}] \\
& -\frac{-2 \xi_{n}}{\left(1+\xi_{n}^{2}\right)^{2}} \times \frac{5}{3\left(1+\xi_{n}^{2}\right)^{3}} \xi_{\mu} \xi_{\nu} \sum_{i<n} R_{i \mu i \nu}^{\partial_{M}}\left(x_{0}\right) \operatorname{tr}[\mathrm{id}] \\
& -\frac{-2 \xi_{n}}{\left(1+\xi_{n}^{2}\right)^{2}} \times \frac{6}{\left(1+\xi_{n}^{2}\right)^{3}} h^{\prime \prime}(0) \xi_{n}^{2} \operatorname{tr}[\mathrm{id}] \\
& -\frac{-2 \xi_{n}}{\left(1+\xi_{n}^{2}\right)^{2}} \times \frac{4}{3\left(1+\xi_{n}^{2}\right)^{4}} \xi_{\mu} \xi_{\nu} \xi_{\gamma} \xi_{\delta} \\
& \times \sum_{\gamma, \delta<n}\left(R_{\mu \nu \nu \delta}^{\partial_{M}}\left(x_{0}\right)+R_{\nu \gamma \mu \delta}^{\partial_{M}}\left(x_{0}\right)\right) \operatorname{tr}[\mathrm{id}] \\
& -\frac{-2 \xi_{n}}{\left(1+\xi_{n}^{2}\right)^{2}} \times \frac{1}{3\left(1+\xi_{n}^{2}\right)^{3}} \xi_{\alpha} \xi_{\beta} \\
& \times \sum_{\alpha, \beta<n}\left(R_{\mu \alpha \nu \beta}^{\partial_{M}}\left(x_{0}\right)+R_{\nu \beta \mu \alpha}^{\partial_{M}}\left(x_{0}\right)\right) \operatorname{tr}[\mathrm{id}] \\
& +\frac{-2 \xi_{n}}{\left(1+\xi_{n}^{2}\right)^{2}} \times \frac{4 h^{\prime \prime}(0)}{\left(1+\xi_{n}^{2}\right)^{4}} \xi_{n}^{2} \operatorname{tr}[\mathrm{id}]+\frac{-2 \xi_{n}}{\left(1+\xi_{n}^{2}\right)^{2}} \\
& \times \frac{h^{\prime \prime}(0)}{\left(1+\xi_{n}^{2}\right)^{3}} \operatorname{tr}[\mathrm{id}]+\frac{-2 \xi_{n}}{\left(1+\xi_{n}^{2}\right)^{2}} \\
& \times \frac{2+3 \xi_{n}+10 \xi_{n}^{2}+12 \xi_{n}^{3}-4 \xi_{n}^{4}+9 \xi_{n}^{5}\left(h^{\prime}(0)\right)^{2} \operatorname{tr}[\mathrm{id}]}{\left(1+\xi_{n}^{2}\right)^{5}}
\end{aligned}
$$

Combining (64), (111), and (123), we obtain

$$
\begin{aligned}
& -i \int_{\left|\xi^{\prime}\right|=1} \int_{-\infty}^{+\infty} \operatorname{trace}\left[\sigma_{-4}\left(D^{-2}\right) \partial_{\xi_{n}} \sigma_{-2}\left(D^{-2}\right)\right] \\
& \quad \times\left(x_{0}\right) \mathrm{d} \xi_{n} \sigma\left(\xi^{\prime}\right) \mathrm{d} x^{\prime} \\
& =-i s\left(x_{0}\right) \Omega_{5} \int_{-\infty}^{+\infty} \frac{4 \xi_{n}}{\left(1+\xi_{n}^{2}\right)^{4}} \mathrm{~d} \xi_{n} \mathrm{~d} x^{\prime} \\
& -i s_{\partial_{M}}\left(x_{0}\right) \Omega_{5} \int_{-\infty}^{+\infty} \frac{56 \xi_{n}}{9\left(1+\xi_{n}^{2}\right)^{5}} \mathrm{~d} \xi_{n} \mathrm{~d} x^{\prime} \\
& -i h^{\prime \prime}(0) \Omega_{5} \int_{-\infty}^{+\infty} \frac{-16 \xi_{n}+16 \xi_{n}^{3}+96 \xi_{n}^{5}}{\left(1+\xi_{n}^{2}\right)^{6}} \mathrm{~d} \xi_{n} \mathrm{~d} x^{\prime} \\
& -i\left(h^{\prime}(0)\right)^{2} \Omega_{5} \int_{-\infty}^{+\infty}\left(\left(-100 \xi_{n}-144 \xi_{n}^{2}-66 \xi_{n}^{3}-576 \xi_{n}^{4}\right.\right. \\
& \left.\quad+1050 \xi_{n}^{5}-432 \xi_{n}^{6}+434 \xi_{n}^{7}\right) \\
& \left.\times\left(3\left(1+\xi_{n}^{2}\right)^{7}\right)^{-1}\right) \mathrm{d} \xi_{n} \mathrm{~d} x^{\prime}
\end{aligned}
$$

$$
\begin{aligned}
& =-\left.i s\left(x_{0}\right) \frac{2 \pi i}{3 !}\left[\frac{4 \xi_{n}}{\left(\xi_{n}+i\right)^{4}}\right]^{(3)}\right|_{\xi_{n}=i} \Omega_{5} \mathrm{~d} x^{\prime} \\
& -\left.i s_{\partial_{M}}\left(x_{0}\right) \frac{2 \pi i}{4 !}\left[\frac{56 \xi_{n}}{9\left(\xi_{n}+i\right)^{5}}\right]^{(4)}\right|_{\xi_{n}=i} \Omega_{5} \mathrm{~d} x^{\prime} \\
& -\left.i h^{\prime \prime}(0) \frac{2 \pi i}{5 !}\left[\frac{-16 \xi_{n}+16 \xi_{n}^{3}+96 \xi_{n}^{5}}{\left(\xi_{n}+i\right)^{6}}\right]^{(5)}\right|_{\xi_{n}=i} \Omega_{5} \mathrm{~d} x^{\prime} \\
& -i\left(h^{\prime}(0)\right)^{2} \frac{2 \pi i}{6 !}\left[\left(-100 \xi_{n}-144 \xi_{n}^{2}-66 \xi_{n}^{3}-576 \xi_{n}^{4}\right.\right. \\
& \left.\quad+1050 \xi_{n}^{5}-432 \xi_{n}^{6}+434 \xi_{n}^{7}\right) \\
& \left.\times\left(3\left(\xi_{n}+i\right)^{7}\right)^{-1}\right]\left.^{(6)}\right|_{\xi_{n}=i} \Omega_{5} \mathrm{~d} x^{\prime} \\
& =3 i\left(h^{\prime}(0)\right)^{2} \pi \Omega_{5} \mathrm{~d} x^{\prime} .
\end{aligned}
$$

Therefore,

$$
\text { Case } \begin{aligned}
15=( & \frac{-1}{4} s\left(x_{0}\right)-\frac{35}{96} s_{\partial_{M}}\left(x_{0}\right)+\left(\frac{343}{192}+\frac{3 i}{2}\right) \\
& \left.\times\left(h^{\prime}(0)\right)^{2}+\frac{13}{16} h^{\prime \prime}(0)\right) \pi \Omega_{5} \mathrm{~d} x^{\prime} .
\end{aligned}
$$

Now, $\Phi$ is the sum of the case $(1,2, \ldots, 15)$, so

$$
\begin{array}{r}
\Phi=\sum_{I=1}^{15} \text { case } I=\left(-\frac{1475}{384}\left(h^{\prime}(0)\right)^{2}+\frac{25}{8} h^{\prime \prime}(0)\right. \\
\left.-\frac{1}{2} s-\frac{77}{192} s_{\partial_{M}}\right) \pi \Omega_{5} \mathrm{~d} x^{\prime} .
\end{array}
$$

Hence, we conclude that, for 7-dimensional compact manifold $M$ with the boundary $\partial M$,

$$
\begin{aligned}
\operatorname{Vol}_{7}^{(2,2)}=\frac{1}{2} \int_{\partial_{M}}( & -\frac{1475}{192}\left(h^{\prime}(0)\right)^{2}+\frac{25}{4} h^{\prime \prime}(0) \\
& \left.-s-\frac{77}{96} s_{\partial_{M}}\right) \pi \Omega_{5} \mathrm{~d} v o l_{\partial_{M}}
\end{aligned}
$$

Next, we recall the Einstein-Hilbert action for manifolds with boundary (see [13] or [14]):

$$
I_{\mathrm{Gr}}=\frac{1}{16 \pi} \int_{M} s \mathrm{~d} \mathrm{vol}_{M}+2 \int_{\partial M} K \mathrm{~d} \mathrm{vol}_{\partial_{M}}:=I_{\mathrm{Gr}, i}+I_{\mathrm{Gr}, b},
$$

where

$$
K=\sum_{1 \leq i, j \leq n-1} K_{i, j} g_{\partial M}^{i, j} ; \quad K_{i, j}=-\Gamma_{i, j}^{n}
$$

and $K_{i, j}$ is the second fundamental form or extrinsic curvature. Take the metric in Section 2; then, by Lemma A.2 in [13], 
we have $K_{i, j}\left(x_{0}\right)=-\Gamma_{i, j}^{n}\left(x_{0}\right)=-(1 / 2) h^{\prime}(0)$ for $i=j<n$; $\Gamma_{s, t}^{i}\left(x_{0}\right)=0$, if $i<n$. For $n=7$, then

$$
K\left(x_{0}\right)=\sum_{i, j} K_{i, j}\left(x_{0}\right) g_{\partial M}^{i, j}\left(x_{0}\right)=\sum_{i=1}^{6} K_{i, i}\left(x_{0}\right)=-\frac{5}{2} h^{\prime}(0) .
$$

So

$$
I_{\mathrm{Gr}, b}=-5 h^{\prime}(0) \mathrm{Vol}_{\partial M} \text {. }
$$

On the other hand, by Proposition 2.10 in [21], we have the following lemma.

Lemma 12. Let $M$ be a 7-dimensional compact manifold with the boundary $\partial M$; then,

$$
s_{M}\left(x_{0}\right)=\frac{3}{2}\left(h^{\prime}(0)\right)^{2}-6 h^{\prime \prime}(0)+s_{\partial_{M}}\left(x_{0}\right) .
$$

Proof. From Proposition 2.10 in [21], let $B=[0,1), b^{2}=$ $\left(1 / h\left(x_{n}\right)\right)$, and $F=\partial_{M}$; we obtain $s_{B}=0$, $\left|\operatorname{grad}_{B} b\right|^{2}=\left(b^{\prime}\right)^{2}$, and

$$
s_{M}\left(x_{0}\right)=12 b^{\prime \prime}\left(x_{0}\right)-30\left(b^{\prime}\left(x_{0}\right)\right)^{2}+s_{\partial_{M}}\left(x_{0}\right) .
$$

By a simple computation, the lemma as follows.

Hence, from (127) and (133), we obtain the following.

Theorem 13. Let $M$ be a 7-dimensional compact manifold with the boundary $\partial M$; then,

$$
\begin{aligned}
& \widetilde{\text { Wres }}\left[\left(\pi^{+} D^{-2}\right)^{2}\right] \\
& =\frac{\pi^{4}}{48} \int_{\partial_{M}}\left(-\frac{47}{2} K^{2}-\left.49 s_{M}\right|_{\partial_{M}}-\frac{77}{4} s_{\partial_{M}}\right) \mathrm{d} \mathrm{vol}_{\partial_{M}} .
\end{aligned}
$$

\section{Conflict of Interests}

The authors declare that there is no conflict of interests regarding the publication of this paper.

\section{Acknowledgments}

This work was supported by NSFC 11271062, NCET-13-0721, and Fok Ying Tong Education Foundation under Grant no. 121003. The authors also thank the referee for his (or her) careful reading and helpful comments.

\section{References}

[1] V. Guillemin, "A new proof of Weyl's formula on the asymptotic distribution of eigenvalues," Advances in Mathematics, vol. 55, no. 2, pp. 131-160, 1985.

[2] M. Wodzicki, "local invariants of spectral asymmetry," Inventiones Mathematicae, vol. 75, no. 1, pp. 143-178, 1995.

[3] M. Adler, "On a trace functional for formal pseudo differential operators and the symplectic structure of the Korteweg-de Vries type equations," Inventiones Mathematicae, vol. 50, no. 3, pp. 219-248, 1978/79.
[4] A. Connes, "Quantized calculus and applications," in Proceedings of the 11th International Congress of Mathematical Physics, pp. 15-36, Internat Press, Cambridge, Mass, USA, 1994.

[5] A. Connes, "The action functinal in Noncommutative geometry," Communications in Mathematical Physics, vol. 117, pp. 673683, 1988.

[6] D. Kastler, "The Dirac operator and gravitation," Communications in Mathematical Physics, vol. 166, no. 3, pp. 633-643, 1995.

[7] W. Kalau and M. Walze, "Gravity, non-commutative geometry and the Wodzicki residue," Journal of Geometry and Physics, vol. 16, no. 4, pp. 327-344, 1995.

[8] T. Ackermann, "A note on the Wodzicki residue," Journal of Geometry and Physics, vol. 20, no. 4, pp. 404-406, 1996.

[9] B. V. Fedosov, F. Golse, E. Leichtnam, and E. Schrohe, "The noncommutative residue for manifolds with boundary," Journal of Functional Analysis, vol. 142, no. 1, pp. 1-31, 1996.

[10] E. Schrohe, "Noncommutative residue, Dixmier's trace, and heat trace expansions on manifolds with boundary," Contemporary Mathematics, vol. 242, pp. 161-186, 1999.

[11] Y. Wang, "Differential forms and the Wodzicki residue for manifolds with boundary," Journal of Geometry and Physics, vol. 56, no. 5, pp. 731-753, 2006.

[12] S. W. Hawking, General Relativity. An Einstein Centenary Survey, S. W. Hawking and W. Israel, Eds., Cambridge University Press, Cambridge, Mass, USA, 1979.

[13] Y. Wang, "Gravity and the noncommutative residue for manifolds with boundary," Letters in Mathematical Physics, vol. 80, no. 1, pp. 37-56, 2007.

[14] Y. Wang, "Lower-dimensional volumes and Kastler-KalauWalze type theorem for manifolds with boundary," Communications in Theoretical Physics, vol. 54, no. 1, pp. 38-42, 2010.

[15] R. Ponge, "Noncommutative Geometry and lower dimensional volumes in Riemannian geometry," Letters in Mathematical Physics, vol. 83, pp. 1-19, 2008.

[16] J. Wang and Y. Wang, "The Kastler-Kalau-Walze type theorem for 6-dimensional manifolds with boundary," http://arxiv.org/abs/1211.6223.

[17] Y. Wang, "A Kastler-KALau-Walze type theorem and the spectral action for perturbations of Dirac operators on manifolds with boundary," Abstract and Applied Analysis, vol. 2014, Article ID 619120, 13 pages, 2014.

[18] J. Wang and Y. Wang, "Noncommutative residue and sub-Dirac operators for foliations," Journal of Mathematical Physics, vol. 54, no. 1, Article ID 012501, 35 pages, 2013.

[19] F. Dobarro and B. Ünal, "Curvature of multiply warped products," Journal of Geometry and Physics, vol. 55, no. 1, pp. 75-106, 2005.

[20] W. Zhang, Local Index Theorem of Atiyah-Singer for Families of Dirac Operators, vol. 1369 of Lecture Notes in Mathematics, Springer, New York, NY, USA, 1989.

[21] Y. Wang, "Multiply warped products with a semisymmetric metric connection," Abstract and Applied Analysis, vol. 2014, Article ID 742371, 12 pages, 2014. 


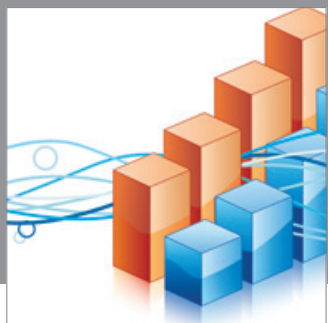

Advances in

Operations Research

mansans

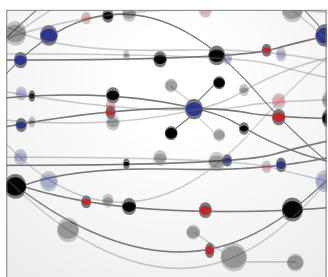

The Scientific World Journal
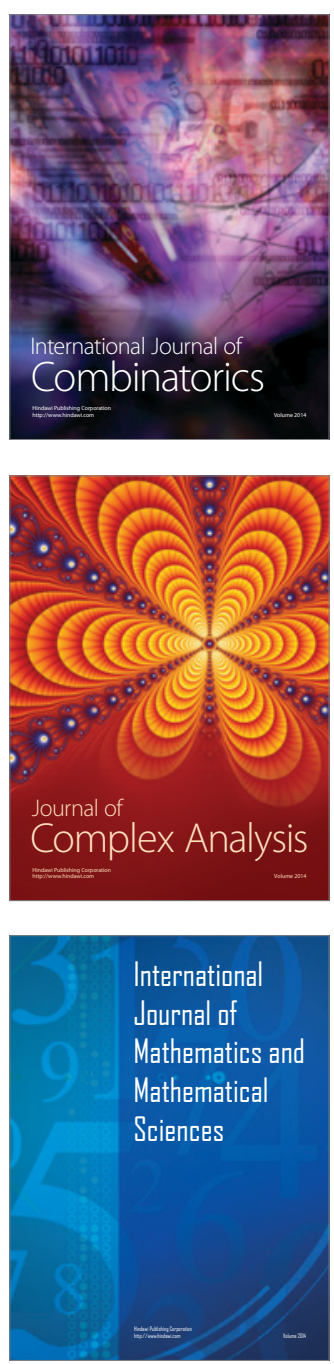
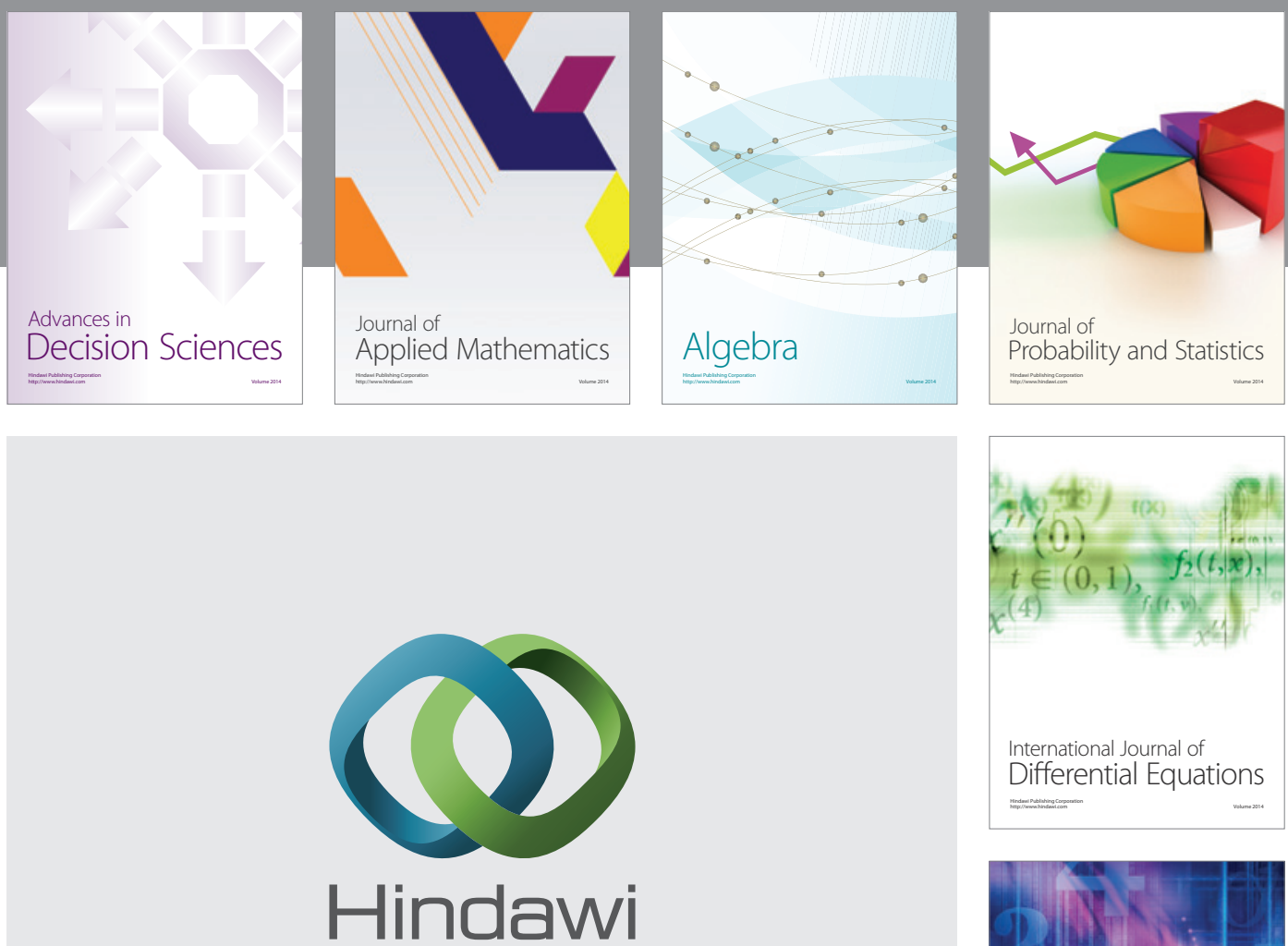

Submit your manuscripts at http://www.hindawi.com
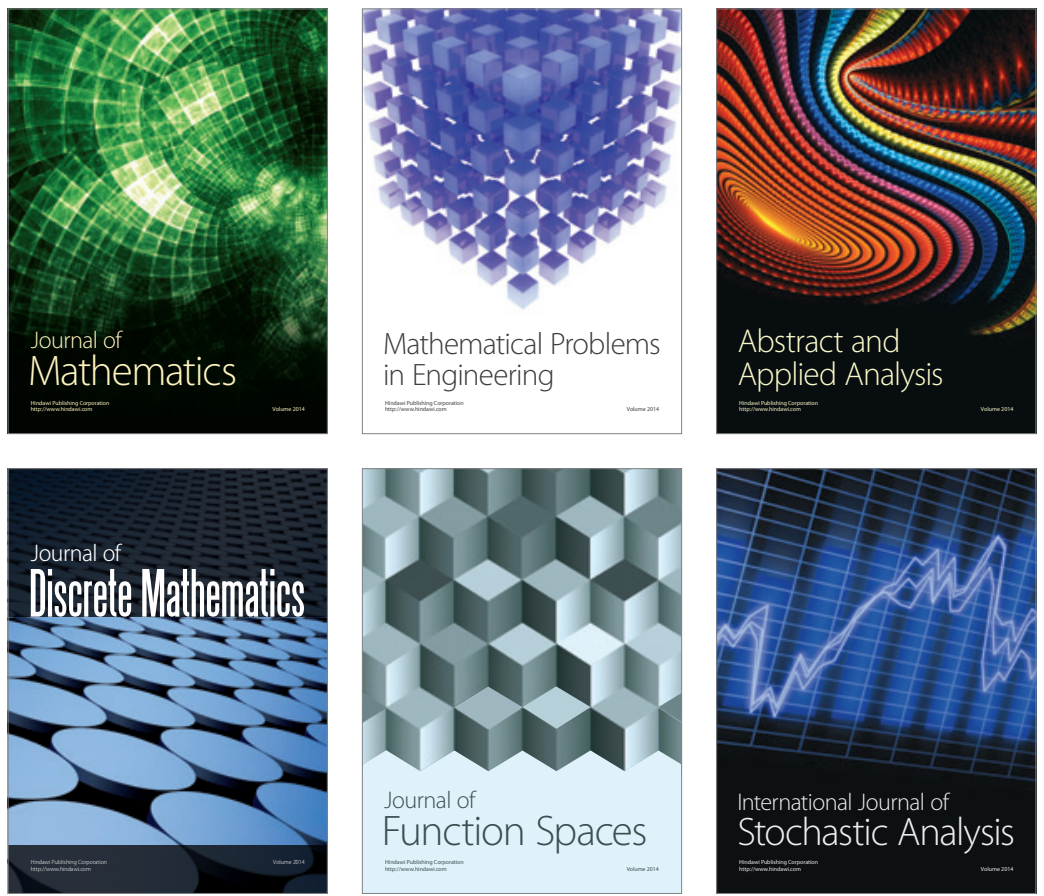

Journal of

Function Spaces

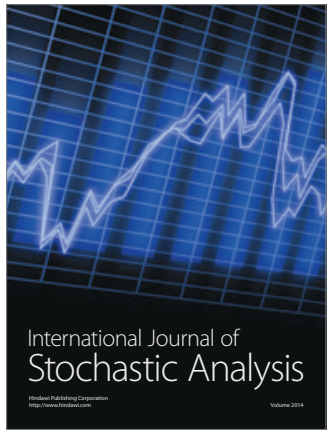

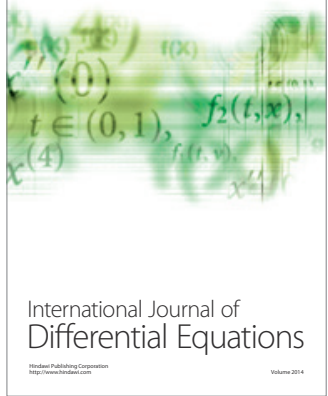
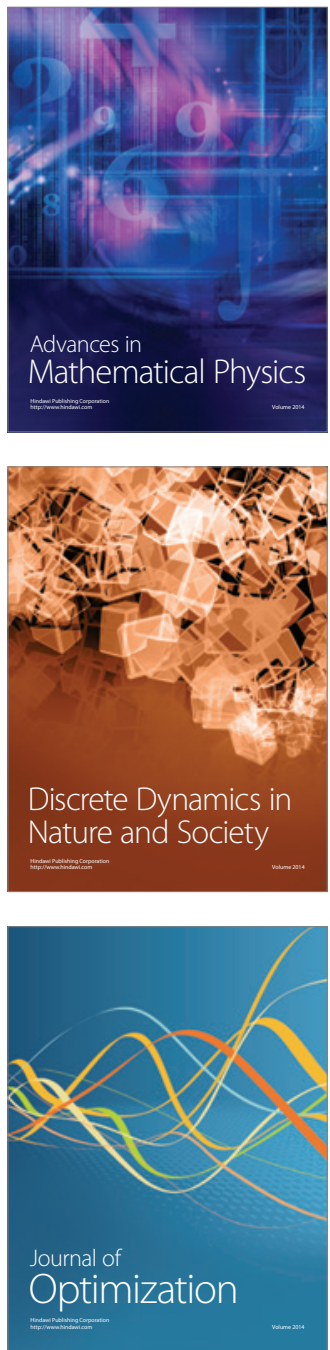